\title{
Hnrnph1 Is A Quantitative Trait Gene for Methamphetamine Sensitivity
}

\author{
Neema Yazdani ${ }^{1,2}$, Clarissa C. Parker ${ }^{3 \mathrm{a} a}$, Ying Shen ${ }^{4 \mathrm{ab}}$, Eric R. Reed ${ }^{1,5}$, Michael A. Guido $^{3}$, \\ Loren A. Kole ${ }^{3}$, Stacey L. Kirkpatrick ${ }^{1}$, Jackie E. Lim ${ }^{3}$, Greta Sokoloff ${ }^{3 \mathrm{ac}}$, Riyan Cheng $^{3 \mathrm{ad}}$, \\ W. Evan Johnson ${ }^{4}$, Abraham A. Palmer ${ }^{6,7}$, Camron D. Bryant ${ }^{1}$ * \\ 1 Laboratory of Addiction Genetics, Department of Pharmacology and Experimental Therapeutics and \\ Department of Psychiatry, Boston University School of Medicine, Boston, Massachusetts, United States of \\ America, 2 NIGMS Ph.D. Program in Biomolecular Pharmacology, Department of Pharmacology and \\ Experimental Therapeutics, Boston University School of Medicine, Massachusetts, United States of America, \\ 3 Department of Human Genetics, The University of Chicago, Chicago, Illinois, United States of America, \\ 4 Division of Computational Biomedicine, Boston University School of Medicine, Boston, Massachusetts, \\ United States of America, 5 Graduate Program in Bioinformatics, Boston University, Boston, Massachusetts, \\ United States of America, 6 Department of Human Genetics, The University of Chicago, Chicago, Illinois, \\ United States of America, 7 Department of Psychiatry and Behavioral Neuroscience, The University of \\ Chicago, Chicago, Illinois, United States of America
}

a Current address: Department of Psychology, Middlebury College, Middlebury, Vermont, United States of America

ab Current address: Howard Hughes Medical Institute and Program in Epithelial Biology, Stanford University School of Medicine, Stanford, California, United States of America

ac Current address: Department of Psychology, University of lowa, lowa City, lowa, United States of America

ad Current address: Plant Sciences, Research School of Biology, Australian National University, Canberra, Australian Capital Territory, Australia

* camron@bu.edu

Editor: Gregory P. Copenhaver, The University of North Carolina at Chapel Hill, UNITED STATES

Received: August 5, 2015

Accepted: November 9, 2015

Published: December 10, 2015

Copyright: @ 2015 Yazdani et al. This is an open access article distributed under the terms of the Creative Commons Attribution License, which permits unrestricted use, distribution, and reproduction in any medium, provided the original author and source are credited.

Data Availability Statement: The F2 data and R code are publicly available on github (https://github. com/wevanjohnson/hnrnph1). The transcriptome dataset, and code for RNA-seq analysis are available via NCBI Gene Expression Omnibus (http://www.ncbi. nlm.nih.gov/geo/query/acc.cgi?token= cxkdoeaudvyhlqt\&acc=GSE66366).

Funding: This study was funded by the National Institutes of Health / National Institute on Drug Abuse grants R00DA029635 (CDB), R01DA039168 (CDB), and R01DA021336 (AAP) as well as the National Institutes of Health / National Human Genome Research Institute grant R01HG005692 (WEJ) and

\section{Abstract}

Psychostimulant addiction is a heritable substance use disorder; however its genetic basis is almost entirely unknown. Quantitative trait locus (QTL) mapping in mice offers a complementary approach to human genome-wide association studies and can facilitate environment control, statistical power, novel gene discovery, and neurobiological mechanisms. We used interval-specific congenic mouse lines carrying various segments of chromosome 11 from the DBA/2J strain on an isogenic C57BL/6J background to positionally clone a $206 \mathrm{~kb}$ QTL $(50,185,512-50,391,845 \mathrm{bp})$ that was causally associated with a reduction in the locomotor stimulant response to methamphetamine (2 mg/kg, i.p.; DBA/2J < C57BL/6J)—a non-contingent, drug-induced behavior that is associated with stimulation of the dopaminergic reward circuitry. This chromosomal region contained only two protein coding genes-heterogeneous nuclear ribonucleoprotein, $\mathrm{H} 1$ (Hnrnph1) and RUN and FYVE domain-containing 1 (Rufy1). Transcriptome analysis via mRNA sequencing in the striatum implicated a neurobiological mechanism involving a reduction in mesolimbic innervation and striatal neurotransmission. For instance, Nr4a2 (nuclear receptor subfamily 4, group A, member 2), a transcription factor crucial for midbrain dopaminergic neuron development, exhibited a 2.1-fold decrease in expression (DBA/2J < C57BL/6J; p $4.2 \times 10-{ }^{15}$ ). Transcription activator-like effector nucleases (TALENs)-mediated introduction of frameshift deletions in the first coding exon of Hnrnph1, but not Rufy1, recapitulated the reduced methamphetamine behavioral response, 
the National Institutes of Health / National Institute of General Medical Sciences T32GM008541 (NY). Funding was also provided by the Burroughs Wellcome Fund Transformative Training Program in Addiction Science \#1011479 (NY). The funders had no role in study design, data collection and analysis, decision to publish, or preparation of the manuscript.

Competing Interests: The authors have declared that no competing interests exist. thus identifying Hnrnph1 as a quantitative trait gene for methamphetamine sensitivity. These results define a novel contribution of Hnrnph 1 to neurobehavioral dysfunction associated with dopaminergic neurotransmission. These findings could have implications for understanding the genetic basis of methamphetamine addiction in humans and the development of novel therapeutics for prevention and treatment of substance abuse and possibly other psychiatric disorders.

\section{Author Summary}

Both genetic and environmental factors can powerfully modulate susceptibility to substance use disorders. Quantitative trait locus (QTL) mapping is an unbiased discoverybased approach that is used to identify novel genetic factors and provide new mechanistic insight into phenotypic variation associated with disease. In this study, we focused on the genetic basis of variation in sensitivity to the acute locomotor stimulant response to methamphetamine which is a behavioral phenotype in rodents that is associated with stimulated dopamine release and activation of the brain reward circuitry involved in addiction. Using brute force monitoring of recombination events associated with changes in behavior, we fortuitously narrowed the genotype-phenotype association down to just two genes that we subsequently targeted using a contemporary genome editing approach. The gene that we validated-Hnrnph1 -is an RNA binding protein that did not have any previously known function in psychostimulant behavior or psychostimulant addiction. Our behavioral data combined with our gene expression results provide a compelling rationale for a new line of investigation regarding Hnrnph1 and its role in neural development and plasticity associated with the addictions and perhaps other dopamine-dependent psychiatric disorders.

\section{Introduction}

Substance use disorders (SUDs) involving psychostimulants such as cocaine and methamphetamine (MA) are heritable; however, their major genetic determinants remain poorly defined [1-4]. In particular, genome-wide association studies (GWAS) of psychostimulant abuse have yet to discover the underlying genetic factors or causal sequence variants. SUDs involve multiple discrete steps including initial use, escalation, withdrawal, and relapse, each of which is believed to have a distinct genetic architecture. Therefore, we and others have used model organisms to explore the genetic basis of intermediate phenotypes, including initial drug sensitivity [5]. Model systems have great potential for studying addiction-relevant intermediate phenotypes [6] because they provide exquisite control over environmental conditions, including exposure to psychostimulants.

Psychostimulants activate the mesocorticolimbic reward circuitry in humans [7] and stimulate locomotor activity in mice [8]. The primary molecular targets of psychostimulants are the membrane-spanning monoaminergic transporters. Amphetamines act as substrates and cause reverse transport and synaptic efflux of dopamine, norepinephrine, and serotonin [9-11]. Sensitivity to the locomotor stimulant response to $\mathrm{MA}$ is heritable and may share a genetic basis with the addictive, neurotoxic, and therapeutic properties of amphetamines [8, 12-15]. More broadly, determining the genetic basis of sensitivity to amphetamines may provide insight into the neurobiology of other conditions involving perturbations in dopaminergic signaling, 
including attention deficit hyperactive disorder (ADHD), schizophrenia, and Parkinson's disease [16]. This hypothesis is supported by our recent identification of a genetic correlation between alleles that increased amphetamine-induced euphoria and alleles that decreased risk of schizophrenia and ADHD [17].

We and others have reported several quantitative trait loci (QTLs) in mice that influence MA sensitivity [12, 18-24]. A distinct advantage of QTL analysis is that chromosomal regions can eventually be mapped to their causal polymorphisms. However, obtaining gene-level and nucleotide-level resolution can be extremely challenging when beginning with a lowly recombinant population such as an $\mathrm{F}_{2}$ cross. A classical approach is to fine map QTLs derived from an $\mathrm{F}_{2}$ cross using successively smaller congenic strains. Whereas this approach is efficient for Mendelian alleles, there are only a few examples in which this approach has been successful in identifying alleles for more complex, polygenic traits, such as histocompatibility [25], substance abuse [26] and depressive-like behavior [27].

In the present study, we fine mapped a QTL on chromosome 11 that modulates methamphetamine sensitivity and that segregates between C57BL/6J (B6) and DBA/2J (D2) inbred strains $[12,20]$. We used interval-specific congenic lines in which successively smaller D2-derived segments were introgressed onto a B6 background [28]. We also conducted transcriptome analysis of brain tissue from a congenic line that captured the QTL for reduced MA sensitivity. Our transcriptome analysis focused on the striatum, which is a brain region important for psychostimulant-induced locomotor activity and reward [29]. We used GeneNetwork [30] and in silico expression QTL (eQTL) analysis of several brain regions to identify cis- and trans-eQTLs that may explain changes in the transcriptome caused by this QTL. Finally, to identify the quantitative trait gene responsible for reduced MA sensitivity, we used transcription activator-like effector nucleases (TALENs) to introduce frameshift deletions in the first coding exon of each positional candidate gene [31].

\section{Results}

\section{Identification of a $206 \mathrm{~kb}$ critical interval for reduced MA sensitivity}

Several genome-wide significant QTLs that influenced MA sensitivity were previously reported in this $\mathrm{B} 6 \times \mathrm{D} 2-\mathrm{F}_{2}$ cross, including QTLs on chromosomes $1,8,9,11,15$, and 16 [20]. Here, we further dissected the chromosome 11 QTL (peak = $50 \mathrm{Mb}$; D2 < B6) into 5 min bins and identified a peak LOD score at 25 min post-MA administration (Fig 1). We then produced intervalspecific congenic lines to fine map this QTL. The genomic intervals $(\mathrm{Mb})$ for the congenic lines and the peak $\mathrm{F}_{2}$-derived QTL are illustrated in Fig $2 \mathrm{~A}$ and the SNP markers that defined the congenic intervals for Lines 1-6 are listed in S1 Table. As shown in Fig 2B-2E, some of the congenic lines captured a QTL that reduced MA sensitivity whereas others did not (see also S2A and S2B Fig and S1 Text). Whether or not a strain captured a QTL is indicated by a + or-sign in Fig 2A.

Congenic Line 4 was the smallest congenic that captured a QTL for reduced MA sensitivity. Therefore, we produced subcongenic lines from Line 4, as shown in Fig 3A. The SNP markers that defined the congenic intervals for Lines $4 \mathrm{a}-4 \mathrm{~h}$ are listed in S2 Table. Production and analysis of these congenic lines was more efficient because the D2-derived allele was dominant. Therefore all lines shown in Fig 3 were heterozygous for the D2-derived congenic interval. Once again, some but not all of the congenic lines captured the QTL inherited from Line 4 (Figs 3B, 3C, 3D and S3, S3 Table and S1 Text). Based on the observation that Line 4b but not $4 \mathrm{c}$ captured the QTL, we were able to define a $206 \mathrm{~kb}$ critical interval (Fig 3E). The first proximal SNP in Lines $4 b$ was rs29424921 and first proximal SNP in Line 4c was rs29442500. The physical location of these SNPs defined the boundaries of the critical interval $(50,185,512$ - 


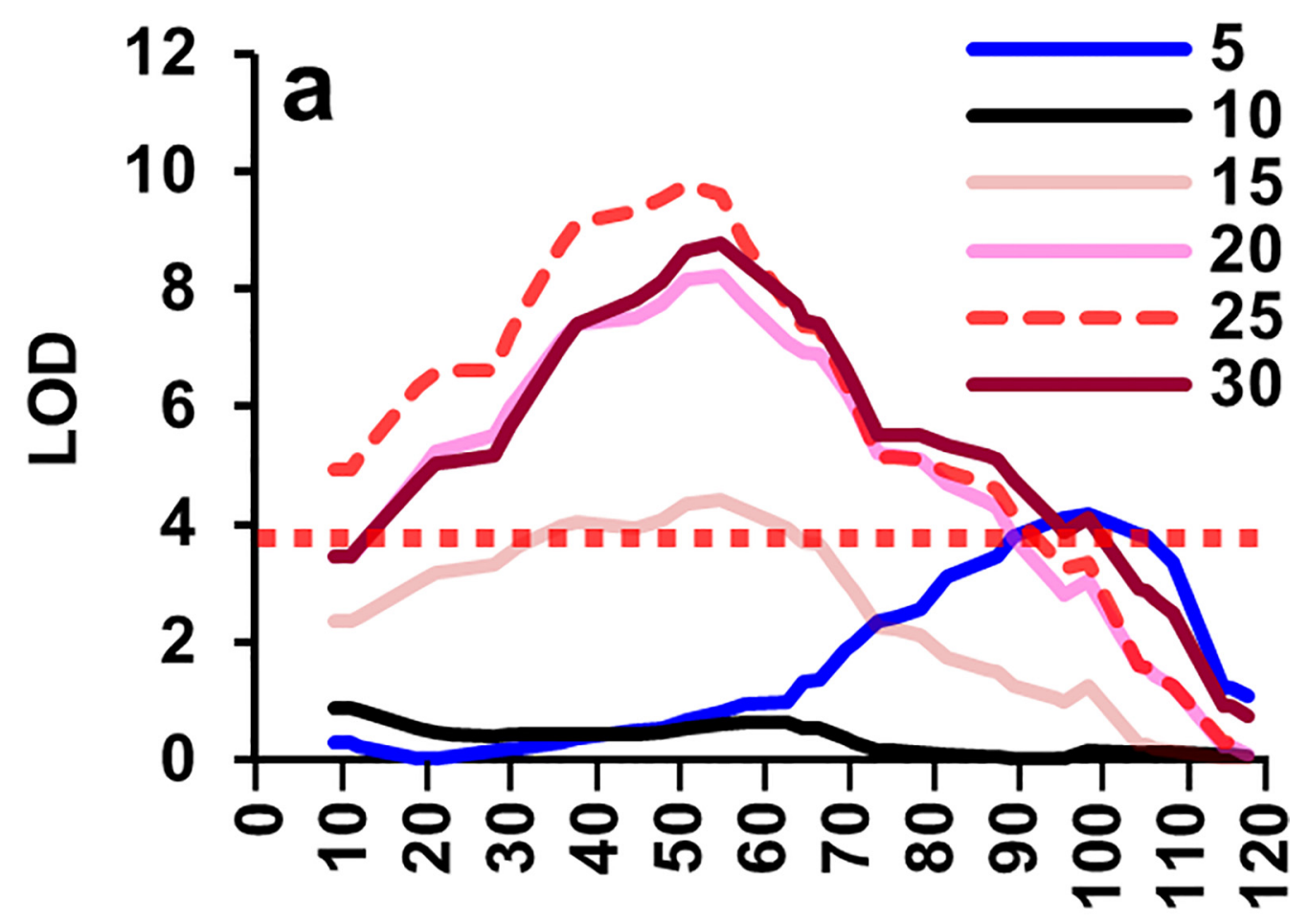

Chromosome 11 position (Mb)

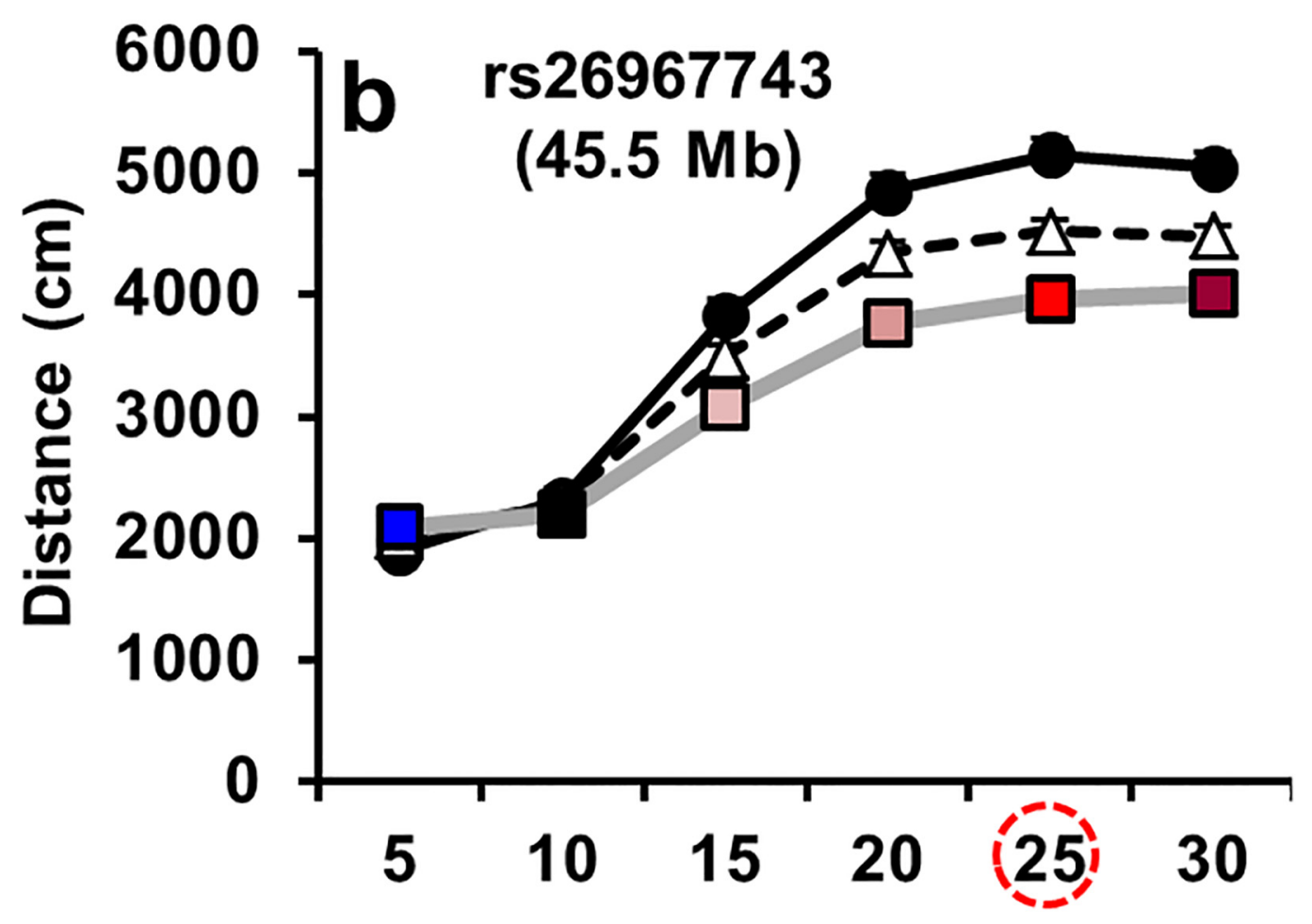

Time post-MA (min) 
Fig 1. QTL for reduced MA sensitivity (D2 < B6) in B6 x D2-F mice. (a): We previously published a genome-wide a significant QTL on chromosome 11 for MA-induced locomotor activity from the same $B 6 \times D 2-F_{2}$ dataset $(N=676)$ that was significant when the data were summed from $15-30$ min and $0-30$ min but not when the data were summed from 0-15 min [20]. To further dissect the time dependency of this locus, we generated LOD scores from the same mice in six, 5-min time bins over $30 \mathrm{~min}$. The x-axis represents the physical distance (Mb) of the marker on chromosome 11 (mm9). The $y$-axis represents the LOD score. The dashed, horizontal line represents the genome-wide significance level derived from 1,000 permutations. The dark blue QTL trace (5 min) denotes a distal locus $(90 \mathrm{Mb})$ in which inheritance of the D2 allele caused an increase in locomotor activity relative to the B6 allele that was most likely not associated with MA treatment (see QTL for Days 1 and 2 in response to saline; S1 Fig; D2 > B6). The remaining red- and pink-shaded QTL traces denote a separate locus $(50 \mathrm{Mb})$ that was specific for MA treatment on Day 3 in which inheritance of the D2 allele caused a decrease in MA-induced locomotor activity. The dashed QTL trace indicates the time bin containing the peak LOD score. (b): The effect plot for the marker nearest the peak LOD score is shown for the six, 5 -min time bins. Data are sorted by genotype for each time bin. The time bin with the most significant LOD score is circled. B6 = homozygous for B6 allele (circles); $\mathrm{H}$ = heterozygous (triangles); D2 = homozygous for D2 allele (colored squares). Data are presented as the mean \pm S.E.M.

doi:10.1371/journal.pgen.1005713.g001

50,391,845 bp; S2 Table). This interval contains only two protein coding genes: Hnrnph1 (heterogeneous nuclear ribonucleoprotein) and Rufyl (RUN and FYVE domain containing 1; Fig 3E and S4 Table).

Using Line $4 c$ to define the distal boundary presumes that our analysis of Line $4 c$ was powerful enough to detect the QTL if it were present. We used data generated from Line $4 \mathrm{~b}$ to estimate the QTL effect size; based on this estimate, a sample size of $\mathrm{N}=25$ per group would be required to achieve $80 \%$ power to detect this QTL in Line $4 \mathrm{c}$. We phenotyped an even larger number of mice from Line $4 \mathrm{c}(\mathrm{N}=30-40$ per genotype), but did not detect the QTL (Fig 3D). Therefore, we can confidently interpret the negative results from Line 4c. Further negative results obtained from five additional subcongenic lines also support the critical interval as defined in Fig 3E (see S3 Fig and S3 Table).

\section{Residual heterozygosity}

Studies of congenic lines can be confounded by residual heterozygosity that lies outside of the congenic region. In order to address this concern, we genotyped individuals from Line 4 subcongenics at 882 SNPs using a SNP genotyping microarray. Although we did identify a single D2-derived SNP on chromosome 3, it was observed both in wild-type and heterozygous congenic mice and was not associated with the locomotor response to MA (see S4 Fig and S1 Text). Based on these results we rejected the possibility that the differences in the congenic lines were due to residual heterozygosity.

\section{Transcriptome of Line 4a}

In an effort to understand the molecular impact of this QTL, we used RNA-seq to identify gene expression differences in the striatum of naïve Line 4a congenics versus their naïve $\mathrm{B} 6$ littermates. We identified between 91 differentially expressed genes with an FDR of $5 \%$ and 174 differentially expressed genes with and FDR of 20\%. The majority of these genes were downregulated in Line 4a (S6 Table). Notably, Nr4a2 (Nurr1) was the most significant, demonstrating a 2.1-fold decrease in expression ( $\mathrm{p}=4.2 \times 10^{-15}$; Fig 4). Decreased Nurr1 expression in Line $4 \mathrm{a}$ was confirmed using qPCR (S5A Fig and S7 Table).

We used the Ingenuity Pathway Analysis (IPA; Ingenuity Systems, Redwood City, CA, USA; www.qiagen.com/ingenuity) software in conjunction with the genes we identified with an FDR of 5\% to explore pathways that were enriched for these genes. The top three canonical pathways that we identified included the neuronal functions Glutamate Receptor Signaling, $\mathrm{G}_{\alpha \mathrm{q}}$ Signaling, and G-Protein Coupled Receptor Signaling (S8 Table). Neither transcriptome nor qPCR analysis detected any significant difference in gene- or exon-level expression of Hnrnph1 or Rufy1 (S5B, S5C and S6 Figs). The most strongly implicated IPA network was, “Cellular Development, Nervous System Development and Function, Behavior". This network consists of several downregulated genes involved in neural development, maintenance, and 


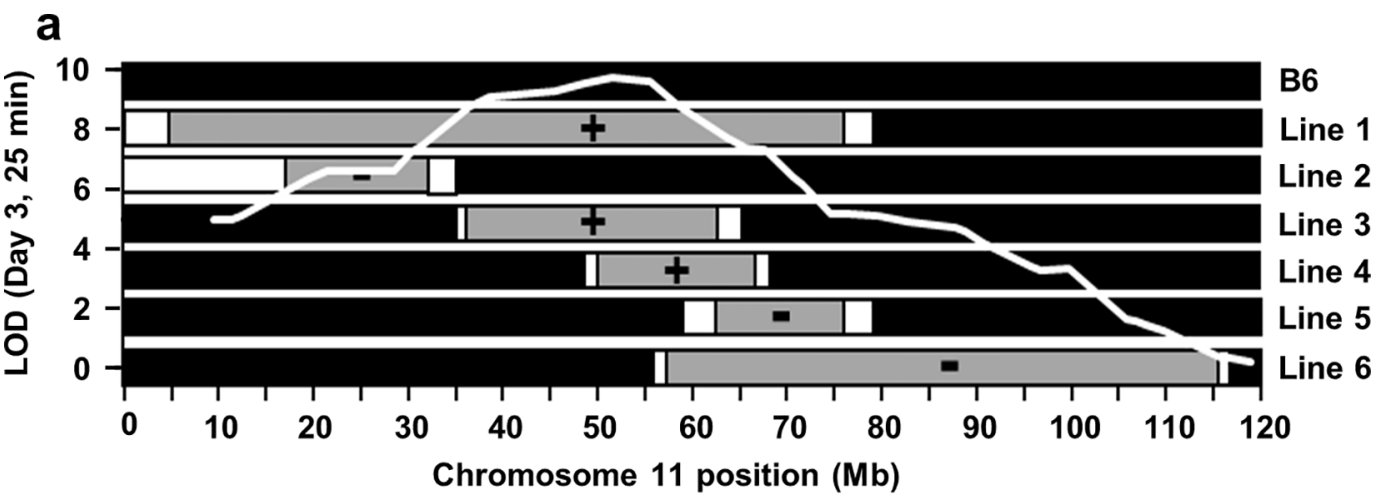

Day 1: SAL
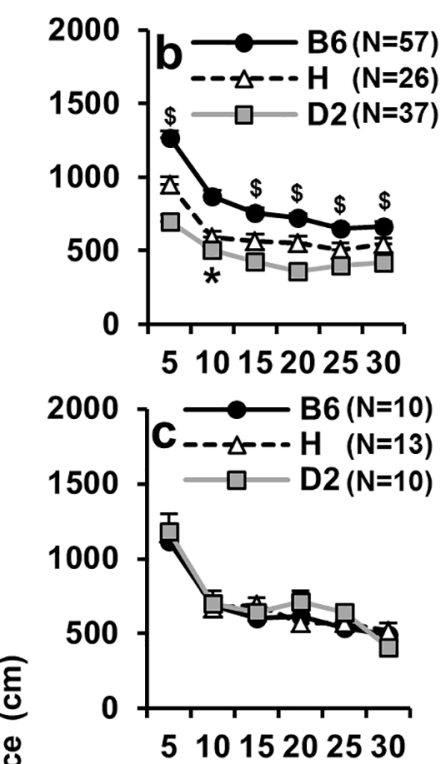

苟

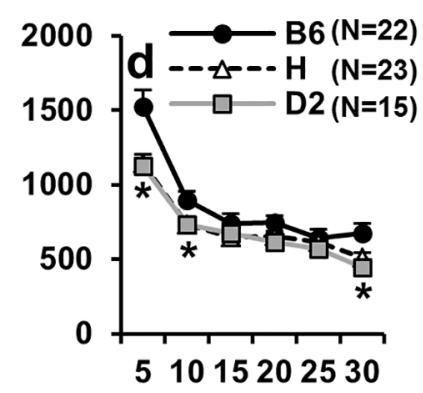

Day 2: SAL

LINE 1
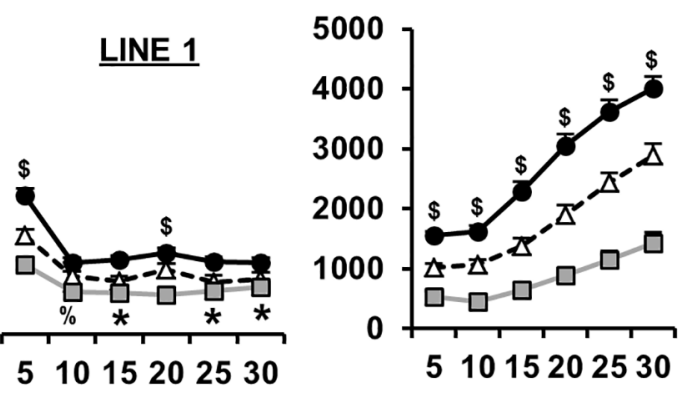

LINE 2

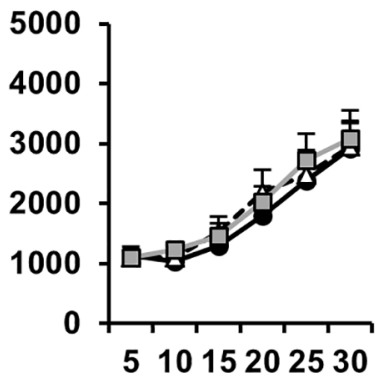

$5 \quad 1015202530$

LINE 3
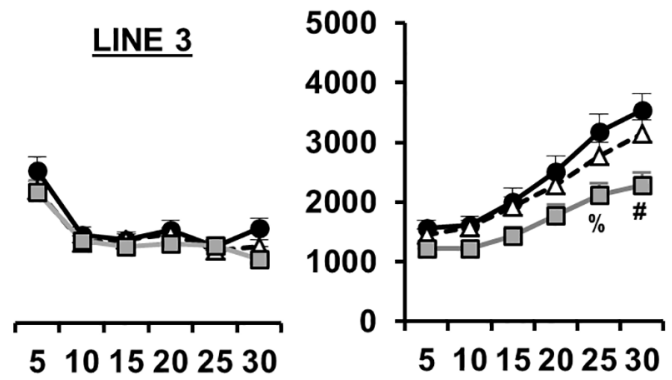

LINE 4
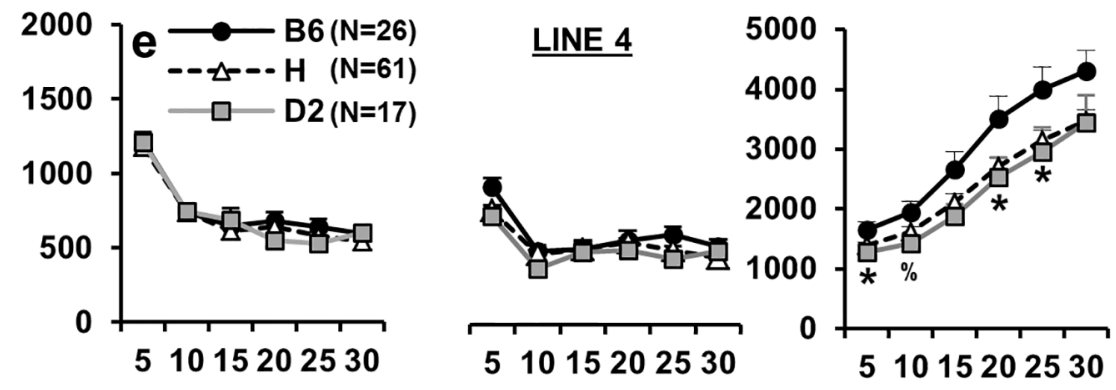

Time post-injection ( $\mathrm{min}$ ) 
Fig 2. Congenic analysis identifies Line 4 for fine mapping. Statistical results are provided in S3 Table and described in the Supplementary Information. (a): Lines 1-6 possessed either one (heterozygous; "H") or two copies (homozygous, "D2") of a chromosome 11 interval from the D2 inbred strain (gray region) on an isogenic $\mathrm{B} 6$ background (black region; denotes the genotype for the rest of the genome). The white regions represent transitional regions that were not genotyped. The x-axis represents the physical position (Mb) of the SNP marker. The SNP markers that were used to genotype Lines $1-6$ are listed in $\mathrm{S} 1 \mathrm{Table}$. The $y$-axis represents the LOD score for the $\mathrm{F}_{2}$-derived QTL that was causally associated with reduced MA sensitivity on Day 3 (D2 < B6; Fig 1; 25 min bin). $(+)=$ congenic line captured the QTL for reduced MA sensitivity on Day $3 .(-)=$ congenic line failed to capture the QTL. (b-e): The three columns represent the phenotypes for Days 1,2, and 3. The four rows represent Lines 1-4. The negative results for Lines 5 and $6(-)$ are shown in S2 Fig and described in S1 Text. "*” indicates a dominant effect of the D2 allele (D2 = H < B6) or H<B6. " $\$$ " indicates an additive effect (D2 < H <6). "\#” indicates a recessive effect (D2 < H = B6). "\%" indicates that B6 and D2 differ from each other but not from $\mathrm{H}$. "\&" indicates that $\mathrm{H}$ and D2 differ from each other but not from B6. Data are represented as the mean \pm S.E.M. $p<0.05$ was considered significant. We estimated the narrow-sense heritability of the $Q T L s\left(h^{2}\right)$ for Line 3 and Line 4 ( $25 \mathrm{~min}$ ) based on the intraclass correlation coefficient using the phenotypic variances from homozygous D2 versus homozygous B6 mice according to the following formula: $\mathrm{h}^{2}=$ (between-genotype variance) / (between-genotype variance + within-genotype variance). For Line $3, \mathrm{~h}^{2}=0.35 ;$ for Line $4, h^{2}=0.08$. Although these $h^{2}$ estimates do not contain confidence intervals, the differences in $h^{2}$ values combined with the different modes of inheritance suggest that Line 3 and Line 4 possess different QTLs.

doi:10.1371/journal.pgen.1005713.g002

signaling (Fig 4), including Bdnf, which was downregulated and connected to several downregulated genes involved in synaptic transmission, including Malat1, the vesicular glutamate transporters VGLUT1 (Slc17a7) and VGLUT2 (Slc17a6), as well as the AMPA-4 receptor subunit (Gria4), alpha-1d adrenergic receptor (Adra1d), and calcium-dependent secretion activator 2 (Cadps2). The top “Diseases and Functions” annotations included Huntington's disease, nervous system coordination, and disorder of basal ganglia (S9 Table), further supporting dysfunction in striatal innervation and signaling. Htt (huntingtin) was the top predicted upstream transcriptional regulator followed by Creb1 (cyclic AMP response element binding protein) which together accounted for 23 (25\%) of the 91 differentially expressed genes (S7 Fig).

Gene Ontology (GO) pathways identified via WebGestalt [32, 33] complemented the IPA results and generally indicate neuronal dysfunction. The top biological process was synaptic transmission and signaling processes, the top molecular functions involved membrane proteins including transporters and g protein-coupled receptors and the top cellular components were associated with neuronal synapses (Table 1).

\section{eQTLs associated with differentially expressed genes in Line 4a}

In order to identify genetic polymorphisms associated with changes in gene expression observed in the congenic region of Line 4a, we used GeneNetwork [30] to identify both cis- and trans-eQTLs that originated from B6/D2 polymorphisms within the Line 4a congenic region (FDR < 20\%; S6 Table). We identified several trans-QTLs caused by SNPs within the Line 4a region, including a link between genetic variation in Hnrnph1 and differential expression of Ipcef1 (Tables 2 and S6) [30], a gene that lies within Oprm1 (mu opioid receptor) and is transcribed in the reverse direction. These observations support the gene expression differences we observed using RNA-seq and indicate that our QTL regulates the expression of numerous other genes outside of the QTL interval.

\section{Recapitulation of the congenic QTL phenotype in mice heterozygous for a frameshift deletion in Hnrnph1, but not Rufy1}

One of the major advantages of genetic analysis in model organisms is the ability to perform experimental manipulations to evaluate observed correlations between genotype and phenotype. We used TALENs to introduce frameshift deletions that resulted in premature stop codons into the first coding exon of each of the two protein coding genes within the $206 \mathrm{~kb}$ critical interval-Hnrnph1 and Rufyl. We identified two founders that were heterozygous for $11 \mathrm{bp}$ and $16 \mathrm{bp}$ frameshift deletions in the first coding exon of Hnrnph1 (Hnrnph1 ${ }^{+/}$; Founders \#28 and \#22; Figs 5A and S8). We did not observe any off-target deletions in the highly homologous 


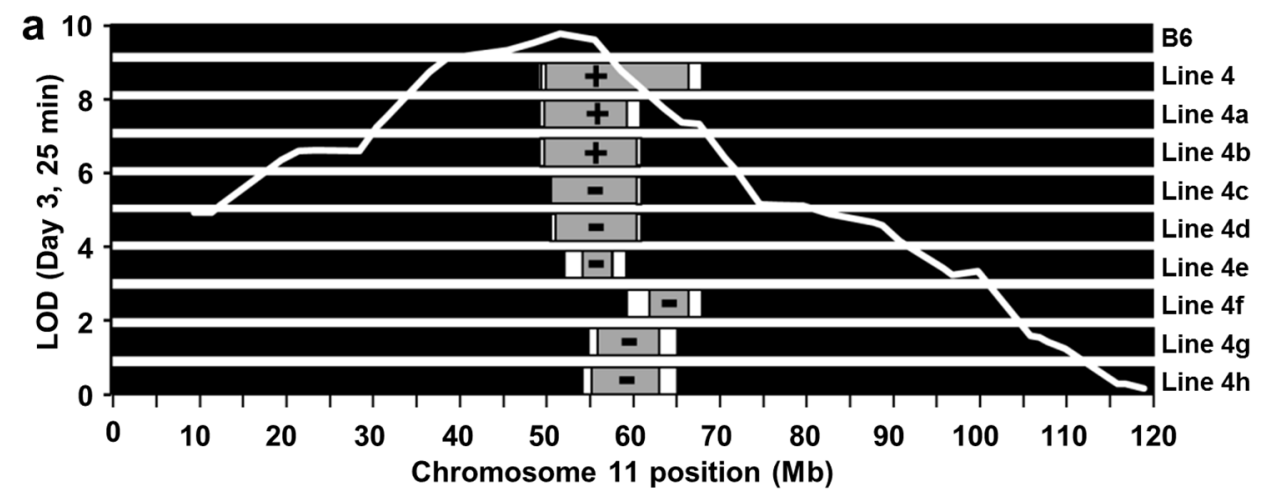
Day 1: SAL Day 2: SAL
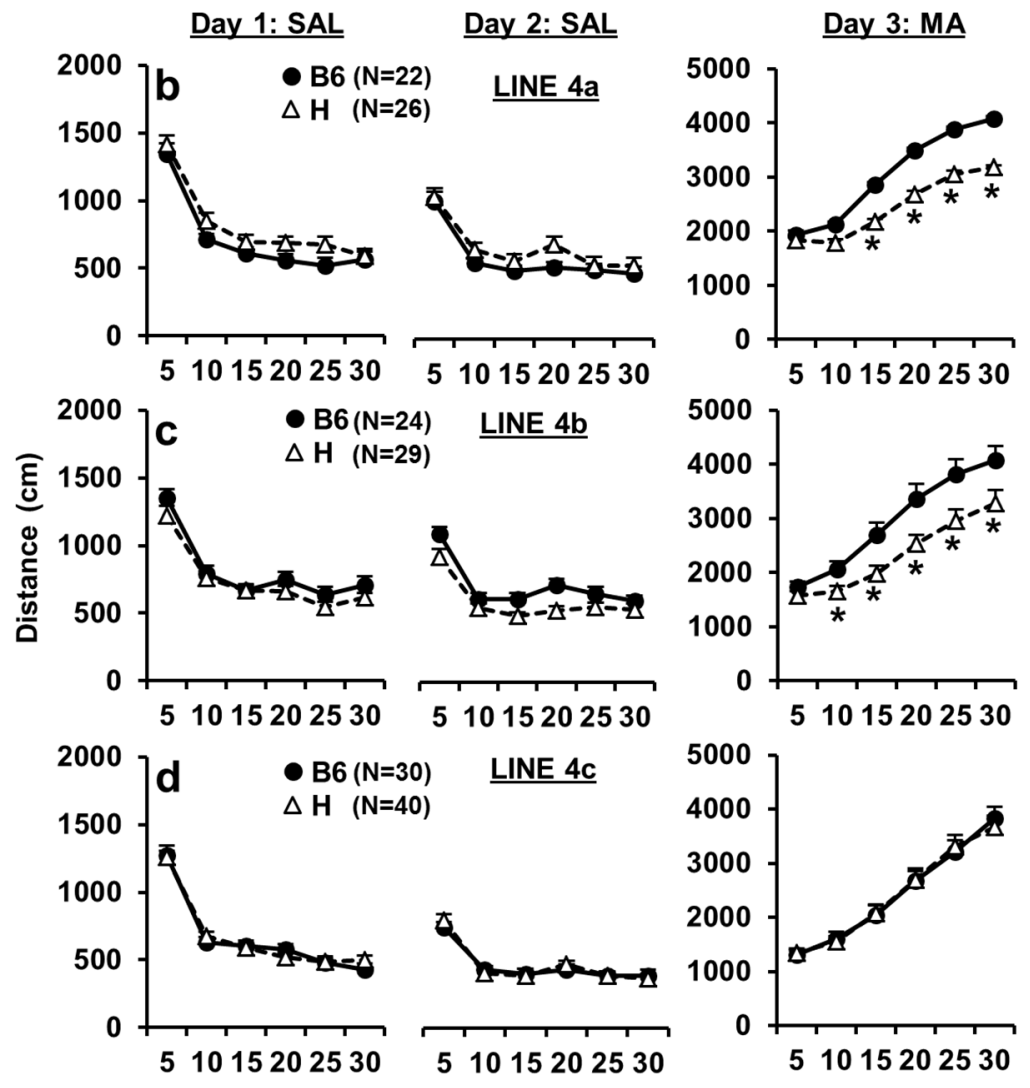

Time post-injection ( $\mathrm{min})$

e Proximal (Build 37)

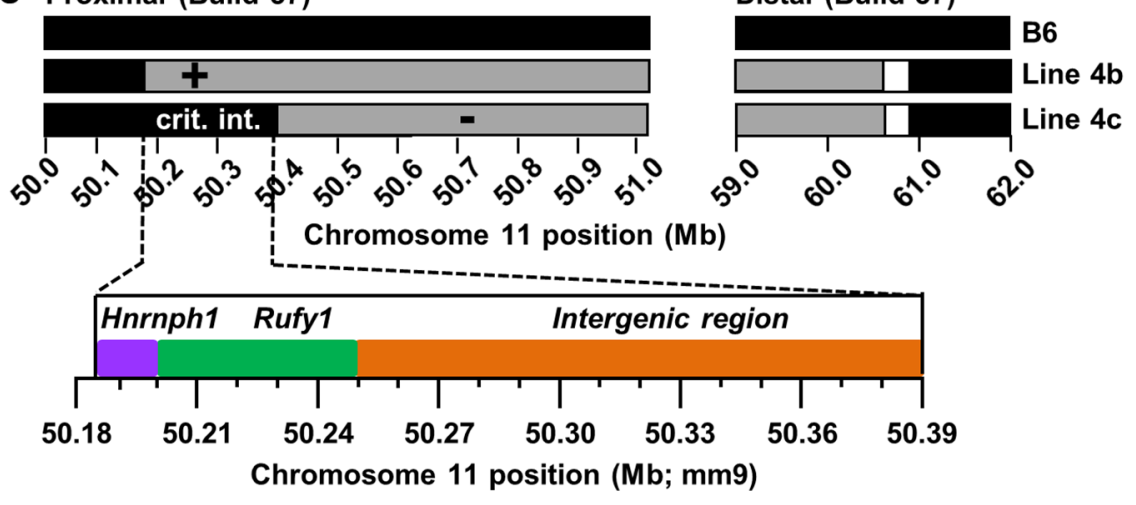


Fig 3. Analysis of subcongenic lines from Line 4 reveals a $206 \mathbf{~ k b}$ critical interval for reduced MA sensitivity. Statistical results are provided in S3 Table and described in the Supplementary Information. (a): Lines $4 \mathrm{a}-4 \mathrm{~h}$ possessed heterozygous $(\mathrm{H})$ intervals of B6 and D2 origin (gray regions) on an isogenic B6 background (black; denotes the genotype for the rest of the genome). The white regions represent transitional regions that were not genotyped. The x-axis represents the physical position (Mb) on chromosome 11. The SNP markers used for genotyping Lines 4a-4h are listed in S2 Table. The y-axis represents the peak LOD score for the $F_{2}$-derived QTL causing reduced MA sensitivity on Day 3 (Fig 1C; 25 min; white QTL trace). $(+)=$ subcongenic line captured the QTL for reduced MA sensitivity. $(-)=$ subcongenic line failed to capture the QTL. (b-d): The three columns represent the phenotypes for Days 1,2 , and 3 . The three rows represent Lines $4 \mathrm{a}-4 \mathrm{c}$. The negative results for Lines $4 \mathrm{~d}-4 \mathrm{~h}(-)$ are shown in S3 Fig and described in S1 Text "**" = significantly different from B6 $(p<0.05)$. Data are represented as the mean \pm S.E.M. (e): The proximal boundary of Line $4 \mathrm{~b}(+)$ and the proximal boundary of Line $4 \mathrm{c}(-)$ define the $206 \mathrm{~Kb}$ critical interval (crit. int.; 50,185,512-50,391,845 bp; mm9; S2 Table) which contains two protein coding genes-Hnrnph1 and Rufy 1 .

doi:10.1371/journal.pgen.1005713.g003

Hnrnph2 gene nor did we observe compensatory change in striatal Hnrnph2 expression (S9 Fig).

Hnrnph $1^{+/-}$mice showed reduced expression of Hnrnph1. When we used qPCR primers that hybridized to DNA sequences that were contained in both wild-type $\left(H n r n p h 1^{+/+}\right)$and Hnrnph $1^{+/-}$mice, there was a significant upregulation of total Hnrnph1 transcript levels in Hnrnph $1^{+/-}$versus Hnrnph $1^{+/+}$mice (Fig 5C and 5D). However, we also used qPCR primers that overlapped the deleted interval and in this case we observed a significant downregulation of Hnrnph $1^{+/+}$transcript levels in Hnrnph $1^{+/-}$mice (Fig 5E). These observations provide functional evidence that the Hnrnph1 frameshift deletion disrupted gene transcription. Similar to Lines 4, $4 \mathrm{a}$ and $4 \mathrm{~b}, H_{n r n p h} 1^{+/-}$mice from Line $\# 28$ and Line \#22 that were derived from Founders \#28 and \#22 both exhibited reduced MA sensitivity (Fig 5F and 5G), thus recapitulating the congenic QTL phenotype. Reduced MA sensitivity was also observed using 30 min behavioral sessions (S10 Fig).

In contrast to Hnrnph1 ${ }^{+/-}$mice, Rufy $1^{+/-}$mice carrying a frameshift deletion (S8 Fig) did not exhibit any difference in behavior (Fig 6). To further support the likelihood of reduced neurobehavioral function in Hnrnph ${ }^{+/}$mice, Hnrnph1 expression is also clearly higher than Rufy1 in the adult brain (S6 Fig; S11 Fig) [34].

To summarize, we observed a significant reduction in MA sensitivity in Hnrnph ${ }^{+/-}$mice, but not $R u f y 1^{+/-}$mice that recapitulated the congenic QTL phenotype, thus identifying Hnrnph1 as a quantitative trait gene for MA sensitivity.

\section{Discussion}

We used positional cloning and gene targeting to identify Hnrnph1 as a novel quantitative trait gene for MA sensitivity. First, we identified a broad, time-dependent QTL on chromosome 11 using an $\mathrm{F}_{2}$ cross between two inbred strains (Fig 1). We then narrowed a QTL from the initial $40 \mathrm{Mb}$ interval to approximately $10 \mathrm{Mb}$ using interval-specific congenic lines (Figs 2, 3, S2 and S3). Further backcrossing yielded a fortuitous recombination event that narrowed a critical interval to just $206 \mathrm{~Kb}$; this region contained only two protein coding genes: Hnrnph1 and Rufy1 (Fig 3E). Striatal transcriptome analysis identified potential neurobiological mechanisms, including a predicted deficit in midbrain dopaminergic neuron development and neurotransmission. The use of GeneNetwork [30] to identify eQTLs associated with our transcriptomic findings provided mechanistic insight, including a trans-QTL that maps to Hnrnph1 that could cause differential expression of Ipcef1 (Table 2; S6 Table). Finally, we took advantage of the power of mouse genetics to create mice heterozygous for a frameshift deletion in either Hnrnph1 or Rufy1. Hnrnph $1^{+/-}$mice but not Rufy $1^{+/-}$mice recapitulated the congenic 


\begin{tabular}{|c|c|c|c|c|c|c|c|}
\hline Gene & Chr/Pos (Mb) & $\underline{P}$ & $\underline{F}$ & $\underline{F}$ & $\underline{F}$ & P Rank & FC Rank \\
\hline Nr4a2 & chr2: 56.96 & $4.2 \mathrm{E}-15$ & -2.1 & $6.0 \mathrm{E}-11$ & 1 & 15 \\
\hline Satb2 & chr1: 56.85 & $1.1 \mathrm{E}-10$ & -1.9 & $3.0 \mathrm{E}-07$ & 5 & 19 \\
\hline Tbr1 & chr2: 61.64 & $1.2 \mathrm{E}-07$ & -1.6 & $1.8 \mathrm{E}-04$ & 9 & 33 \\
\hline Slc8a1 & chr17: 81.77 & $2.8 \mathrm{E}-07$ & -1.5 & $3.3 \mathrm{E}-04$ & 12 & 46 \\
\hline Adra1d & chr2: 131.37 & $2.4 \mathrm{E}-06$ & -1.9 & $1.7 \mathrm{E}-03$ & 20 & 21 \\
\hline Bmp3 & chr5: 99.28 & $9.3 \mathrm{E}-06$ & -2.0 & $4.3 \mathrm{E}-03$ & 31 & 16 \\
\hline Neurod6 & chr6: 55.63 & $1.0 \mathrm{E}-05$ & -1.7 & $4.5 \mathrm{E}-03$ & 32 & 28 \\
\hline Gfra2 & chr14: 71.34 & $1.7 \mathrm{E}-05$ & -1.4 & $6.5 \mathrm{E}-03$ & 36 & 55 \\
\hline Bdnf & chr2: 109.56 & $2.3 \mathrm{E}-05$ & -1.7 & $7.8 \mathrm{E}-03$ & 41 & 30 \\
\hline Satb1 & chr17: 51.88 & $3.1 \mathrm{E}-05$ & -1.3 & 0.00942 & 46 & 75 \\
\hline Gria4 & chr9: 4.42 & $4.3 \mathrm{E}-05$ & -1.3 & $1.2 \mathrm{E}-02$ & 52 & 77 \\
\hline Cadps2 & chr6: 23.21 & $5.0 \mathrm{E}-05$ & -1.4 & $1.3 \mathrm{E}-02$ & 54 & 62 \\
\hline Crym & chr7: 127.34 & $5.7 \mathrm{E}-05$ & 1.4 & $1.4 \mathrm{E}-02$ & 57 & 66 \\
\hline Wfs1 & chr5: 37.35 & $7.6 \mathrm{E}-05$ & 1.4 & $1.7 \mathrm{E}-02$ & 64 & 65 \\
\hline Dlk1 & chr12: 110.69 & $8.2 \mathrm{E}-05$ & 2.4 & $1.7 \mathrm{E}-02$ & 67 & 8 \\
\hline Ets2 & chr16: 95.92 & $1.0 \mathrm{E}-04$ & 1.3 & $2.1 \mathrm{E}-02$ & 68 & 79 \\
\hline Malat1 & chr19: 5.80 & $1.2 \mathrm{E}-04$ & -1.6 & $2.3 \mathrm{E}-02$ & 71 & 37 \\
\hline Elavl2 & chr4: 90.93 & $2.1 \mathrm{E}-04$ & -1.3 & $3.7 \mathrm{E}-02$ & 79 & 83 \\
\hline Slc17a7 & chr7: 52.42 & $2.7 \mathrm{E}-04$ & -1.4 & $4.5 \mathrm{E}-02$ & 85 & 59 \\
\hline Slc17a6 & chr7: 58.88 & $3.1 \mathrm{E}-04$ & -3.0 & $4.9 \mathrm{E}-02$ & 89 & 3 \\
\hline
\end{tabular}

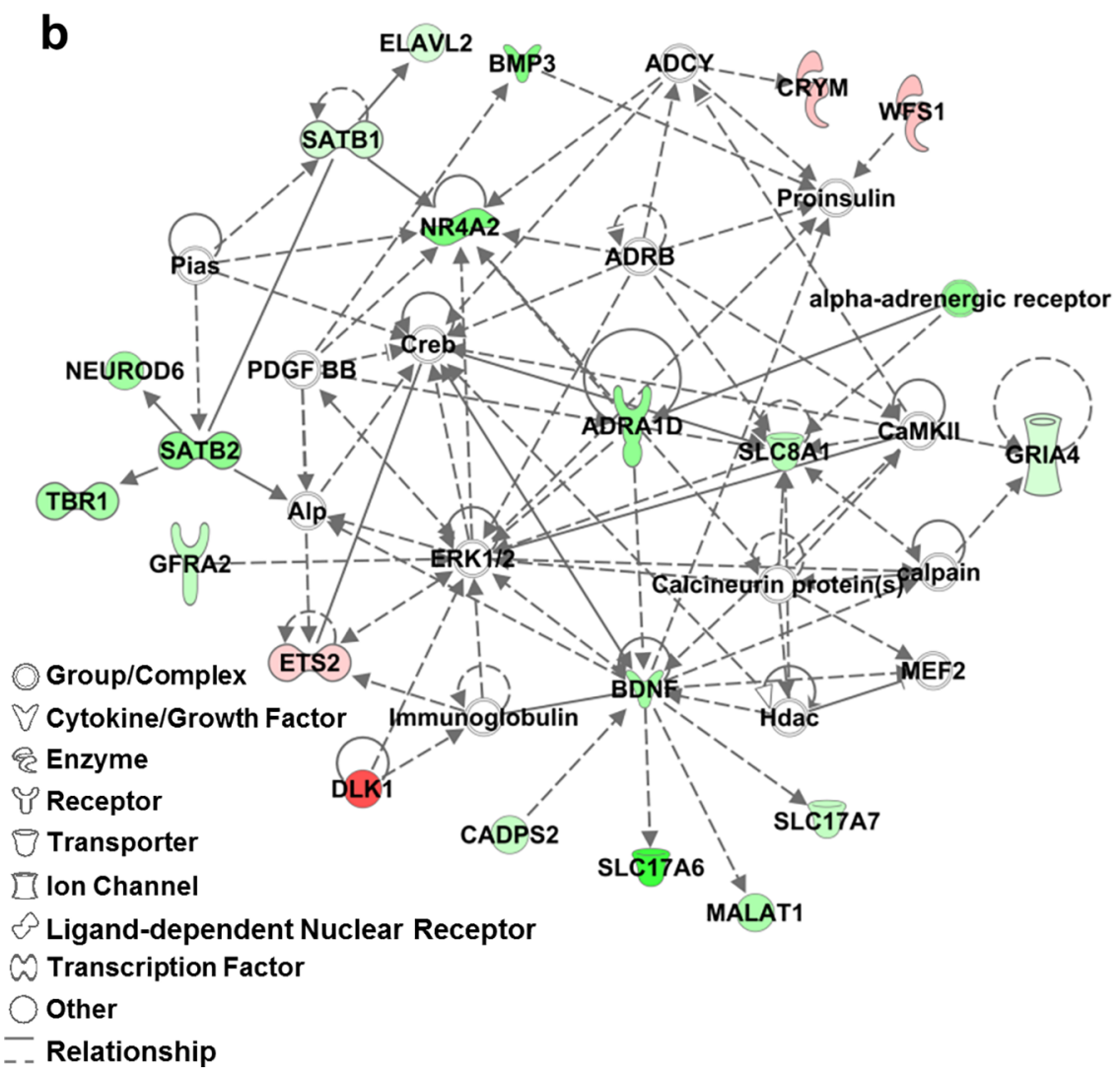


Fig 4. Transcriptome analysis of Line 4a identifies "Cellular Development, Nervous System Development and Function, Behavior" as the top IPA network. (a, b): 17 downregulated genes (green) and four upregulated genes (red) were identified in the IPA network (S10 Table). Genes in the network diagram that lack any color were included by the IPA algorithm to facilitate connectivity. Chromosome and position (Chr/Pos; $\mathrm{mm}$ ) of each gene is shown. $\mathrm{P}=\mathrm{p}$-value of differential expression in Line 4a; $\mathrm{FC}=$ foldchange in expression; FDR = false discovery rate $(<0.05 ; 5 \%), P$ Rank = rank in $p$-value $(\# 1=$ lowest $p$-value out of 91 genes); FC Rank = rank in fold-change (\#1 = largest fold-change out of 91 genes).

doi:10.1371/journal.pgen.1005713.g004

QTL phenotype, providing direct evidence that Hnrnph1 is a quantitative trait gene for MA sensitivity (Figs 5 and 6).

QTL mapping studies of rodent behavior have rarely provided strong evidence for causal quantitative trait genes $[26,27,35]$. We began pursuing this QTL more than a decade ago, when the difficulty of such projects was widely underestimated. A key limitation of our initial mapping strategy was the use of an $\mathrm{F}_{2}$ cross, in which extensive linkage disequilibrium created large haplotype blocks, resulting in the identification of very broad QTLs. Combining low

Table 1. WebGestalt-Gene Onotology (GO) analysis of differentially expressed genes in the striatum of Line 4a. GO enrichment analysis of our gene list (91 genes, FDR < 5\%) was performed using a hypergenometric statistical procedure and multiple testing adjustment (Adj P). A minimum of two genes was required per category.

\begin{tabular}{|c|c|c|c|c|}
\hline Biological Process & GO ID & $\mathbf{P}$ & Adj P & \# of Genes \\
\hline Synaptic transmission & 0007268 & $6.40 \mathrm{E}-12$ & 2.07E-09 & 16 \\
\hline Multicellular organismal signaling & 0035637 & 3.36E-12 & 2.07E-09 & 18 \\
\hline Transmission of nerve impulse & 0019226 & $1.84 \mathrm{E}-11$ & 3.97E-09 & 17 \\
\hline Cell-cell signaling & 0007267 & 1.09E-09 & 1.77E-07 & 17 \\
\hline Single-organism process & 0044699 & $1.91 \mathrm{E}-08$ & 2.48E-06 & 54 \\
\hline Multicellular organismal process & 0032501 & $5.60 \mathrm{E}-07$ & 4.54E-05 & 43 \\
\hline Biological regulation & 0065007 & $5.46 \mathrm{E}-07$ & 4.54E-05 & 57 \\
\hline Single-multicellular organism process & 0044707 & $5.25 \mathrm{E}-07$ & 4.54E-05 & 43 \\
\hline Single organism signaling & 0044700 & $1.25 \mathrm{E}-06$ & 8.10E-05 & 39 \\
\hline Signaling & 0023052 & $1.25 \mathrm{E}-06$ & 8.10E-05 & 39 \\
\hline Molecular Function & GO ID & $\mathbf{P}$ & Adj P & \# of Genes \\
\hline Transporter activity & 0005215 & $1.74 \mathrm{E}-06$ & 2.00E-04 & 16 \\
\hline Transmembrane transporter activity & 0022857 & $1.59 \mathrm{E}-05$ & 1.10E-03 & 13 \\
\hline Secondary active transmembrane transporter activity & 0015291 & 4.94E-05 & $1.80 \mathrm{E}-03$ & 6 \\
\hline Alpha1-adrenergic receptor activity & 0004937 & 4.10E-05 & $1.80 \mathrm{E}-03$ & 2 \\
\hline Substrate-specific transporter activity & 0022892 & $1.00 \mathrm{E}-04$ & 2.40E-03 & 12 \\
\hline Substrate-specific transmembrane transporter activity & 0022891 & $1.00 \mathrm{E}-04$ & 2.40E-03 & 11 \\
\hline Anion transmembrane transporter activity & 0008509 & $2.00 \mathrm{E}-04$ & $3.60 \mathrm{E}-03$ & 6 \\
\hline Transmembrane transporter activity & 0015075 & 4.00E-04 & $6.30 \mathrm{E}-03$ & 10 \\
\hline Adrenergic receptor activity & 0004935 & 5.00E-04 & $6.60 \mathrm{E}-03$ & 2 \\
\hline Cellular Component & GO ID & $\mathbf{P}$ & Adj $P$ & \# of Genes \\
\hline Cell junction & 0030054 & 8.26E-08 & 9.17E-06 & 15 \\
\hline Synapse & 0045202 & 1.07E-06 & 5.94E-05 & 12 \\
\hline Plasma membrane & 0005886 & 3.63E-06 & $1.00 \mathrm{E}-04$ & 31 \\
\hline Cell periphery & 0071944 & 6.17E-06 & 2.00E-04 & 31 \\
\hline Synapse part & 0044456 & 1.87E-05 & $4.00 E-04$ & 9 \\
\hline Cell part & 0005623 & $2.00 \mathrm{E}-04$ & $3.20 \mathrm{E}-03$ & 66 \\
\hline Neuron spine & 0044309 & 4.00E-04 & $4.90 \mathrm{E}-03$ & 5 \\
\hline Dendritic spine & 0043197 & 4.00E-04 & 4.90E-03 & 5 \\
\hline Postsynaptic membrane & 0045211 & $6.00 \mathrm{E}-04$ & 6.70E-03 & 5 \\
\hline
\end{tabular}

doi:10.1371/journal.pgen.1005713.t001 
Table 2. Differentially expressed genes in Line 4a (FDR < 20\%) that possessed cis- or trans-eQTLs in GeneNetwork (GN). Differentially expressed genes (DEGs) are shown from our striatal RNA-seq dataset (FDR $<20 \%$ ) that possess known eQTLs from GeneNetwork caused by genetic variation within the Line 4a locus (chromosome 11: 50-60 Mb). With regard to DEGs from our dataset: Chr/Pos = chromosome and position of each DEG; FC = foldchange; $P=p$-value; $Q=q$-value. With regard to eQTLs identified in GeneNetwork: The GeneNetwork genes associated with differential expression of DEGs from our dataset are listed [LRS $\geq 13.8(\mathrm{LOD} \geq 3)$ ]; NAC = nucleus accumbens; $\mathrm{Str}=$ striatum; NCTX = neocortex; PFC = prefrontal cortex; $\mathrm{HC}=$ hippocampus; $\mathrm{LRS}$ = likelihood ratio statistic; $\mathrm{GN}=$ GeneNetwork. eQTLs were identified from the following datasets: UTHSC Hippocampus Illumina v6.1 All Combined (Nov12) RankInv Database; Hippocampus Consortium M430v2 (Jun06) PDNN Database UTHSC Hippocampus Illumina v6.1 NON (Sep09) RankInv Database; Hippocampus Consortium M430v2 (Jun06) RMA Database; BIDMC-UTHSC Dev Neocortex P3 ILMv6.2 (Nov11) RankInv Database; BIDMC-UTHSC Dev Neocortex P14 ILMv6.2 (Nov11) RankInv Database; HQF BXD Neocortex ILM6v1.1 (Dec10v2) RankInv Database; HQF BXD Neocortex ILM6v1.1 (Feb08) Ranklnv Database; VCU BXD NAc Sal M430 2.0 (Oct07) RMA Database; HQF Striatum Affy Mouse Exon 1.0ST Gene Level (Dec09) RMA Database; HQF BXD Striatum ILM6.1 (Dec10v2) RankInv Database; HBP Rosen Striatum M430V2 (Apr05) RMA Clean Database

\begin{tabular}{|c|c|c|c|c|c|c|c|c|}
\hline $\begin{array}{l}\text { Gene ID for DEG } \\
\text { (RNA-seq) }\end{array}$ & Gene name (RNA-seq) & $\begin{array}{l}\text { Chr/Pos of } \\
\text { DEG (Mb) }\end{array}$ & $\begin{array}{l}\log _{2} F C \text { of } \\
\operatorname{DEG}( \pm F C)\end{array}$ & $\begin{array}{l}\text { P-value } \\
\text { of DEG }\end{array}$ & $\begin{array}{l}\text { FDR of } \\
\text { DEG }\end{array}$ & $\begin{array}{l}\text { Associated GeneNetwork genes } \\
\text { within Line } 4 \text { a region }\end{array}$ & $\begin{array}{l}\text { eQTL } \\
\text { LRS }\end{array}$ & $\begin{array}{l}\text { Brain } \\
\text { Region }\end{array}$ \\
\hline Slc8a1 & $\begin{array}{l}\text { solute carrier family } 8 \\
\text { (sodium/calcium exchanger), } \\
\text { member } 1\end{array}$ & $17: 81.77$ & $-0.59(-1.50)$ & $2.9 \times 10^{-7}$ & $3.3 \times 10^{-4}$ & Olfr51 (50.8 Mb) & 19.1 & NTCX \\
\hline Satb1 & $\begin{array}{l}\text { special AT-rich sequence } \\
\text { binding protein } 1\end{array}$ & $\underline{17: 51.87}$ & $-0.35(-1.27)$ & $3.1 \times 10^{-5}$ & $9.4 \times 10^{-3}$ & B130040O20Rik (49.8 Mb) & 18.2 & NCTX \\
\hline Obscn & obscurin & $\underline{11: 50.89}$ & $1.23(+2.34)$ & $1.9 \times 10^{-5}$ & 0.01 & $\begin{array}{l}\text { 2610507I01Rik,Mrpl55, } \\
\text { D130047N11Rik,Gja12,Guk1, } \\
\text { 2810021J22Rik (50-59 Mb) }\end{array}$ & $20-82$ & $\begin{array}{l}\text { NAc, Str, } \\
\text { NCTX, PFC, } \\
\text { Hipp }\end{array}$ \\
\hline Megf11 & multiple EGF-like-domains 11 & 9: 64.23 & $-0.46(-1.38)$ & $4.5 \times 10^{-5}$ & 0.01 & Mprip (59.5 Mb),Tom1/2 (60.0 Mb) & $\begin{array}{l}14.3 \\
14.4\end{array}$ & NAc, NCTX \\
\hline Malat1 & $\begin{array}{l}\text { metastasis associated lung } \\
\text { adenocarcinoma transcript } 1\end{array}$ & 19:5.79 & $-0.68(1.60)$ & $1.2 \times 10^{-4}$ & 0.023 & I/3 (54.0 Mb) & 15.1 & NCTX \\
\hline$M k x$ & mohawk homeobox & $\underline{18: 6.93}$ & $-0.47(-1.38)$ & $5.1 \times 10^{-4}$ & 0.07 & Olfr323 (58.4 Mb) & 16.0 & NCTX \\
\hline Hs3st2 & $\begin{array}{l}\text { heparan sulfate } 3-\mathrm{O}- \\
\text { sulfotransferase } 2\end{array}$ & 7:128.53 & $-0.52(-1.43)$ & $8.5 \times 10^{-4}$ & 0.11 & Cops3 (59.6 Mb) & 14.4 & PFC \\
\hline Ipcef1 & $\begin{array}{l}\text { interaction protein for } \\
\text { cytohesin exchange factors } 1\end{array}$ & $\underline{10: 3.37}$ & $-0.57(-1.48)$ & $1.1 \times 10^{-3}$ & 0.12 & Hnrnph1 (50.2 Mb), G3bp1 (55.3 Mb) & $\begin{array}{l}15.1 \\
18.8\end{array}$ & NCTX \\
\hline Tgm2 & $\begin{array}{l}\text { transglutaminase } 2, \mathrm{C} \\
\text { polypeptide }\end{array}$ & $\underline{2}: 157.95$ & $0.46(+1.37)$ & $1.4 \times 10^{-3}$ & 0.15 & N4bp3 (51.5 Mb) & 15.7 & PFC \\
\hline 9230009I02Rik & & $\underline{11: 50.89}$ & $-0.94(-1.92)$ & $1.6 \times 10^{-3}$ & 0.16 & $\begin{array}{l}\text { Agxt2/2,D11Ertd497e, Col23a1, } \\
\text { Hnrpab,Lyrm7, G3bp1,Clk4,Damts2, } \\
\text { Gria1,Zfp354a (51-57 Mb) }\end{array}$ & $17-66$ & NCTX \\
\hline Ubash $3 b$ & $\begin{array}{l}\text { ubiquitin associated and } \mathrm{SH} 3 \\
\text { domain containing, B }\end{array}$ & $9: 40.82$ & $-0.61(-1.54)$ & $1.7 \times 10^{-3}$ & 0.17 & Zfp2 (50.7 Mb) & 14.9 & $\mathrm{HC}$ \\
\hline Ablim2 & actin-binding LIM protein 2 & $5: 36.10$ & $0.21(+1.16)$ & $2.4 \times 10^{-3}$ & 0.20 & Olfr54 (36.2 Mb) & 14 & Str \\
\hline
\end{tabular}

doi:10.1371/journal.pgen.1005713.t002

resolution and high resolution QTL mapping in congenic lines revealed a more complex genetic architecture, indicating that Hnrnph1 is not the only causal gene within the $\mathrm{F}_{2}$ interval that underlies the QTL. Inheritance of two copies of the D2 segment enhanced the heterozygous phenotype in Line 1, yet had no further effect once the size of the segment was reduced following the creation of Line 4 (Fig $2 \mathrm{~B}$ and 2E). We interpret this observation to suggest that Line 1 contains an additional, recessive QTL within the 35-50 Mb region of Line 3 that could summate with the Line 4 QTL to produce the larger effect size. This $35-50 \mathrm{Mb}$ region could be fine-mapped to the causal genetic factor by introducing additional recombination events into Line 3. This detailed level of insight into the genetic architecture of a single large-effect QTL could only be made possible by employing a sufficiently powered phenotypic analysis of interval-specific congenic lines. Thus, a key to our success in identifying a single gene was the fact that while the QTL originally identified in the $\mathrm{F}_{2}$ cross was likely the product of multiple smaller QTLs, we were able to capture one major QTL in Line 4 and in subcongenic lines which appears to correspond to a single quantitative trait gene that we have now identified as Hnrnph1.

Transcriptome analysis of Line 4a supports a neurodevelopmental mechanism by which the QTL regulates MA sensitivity. Nr4a2 (a.k.a. Nurr1) was the top downregulated gene and codes 


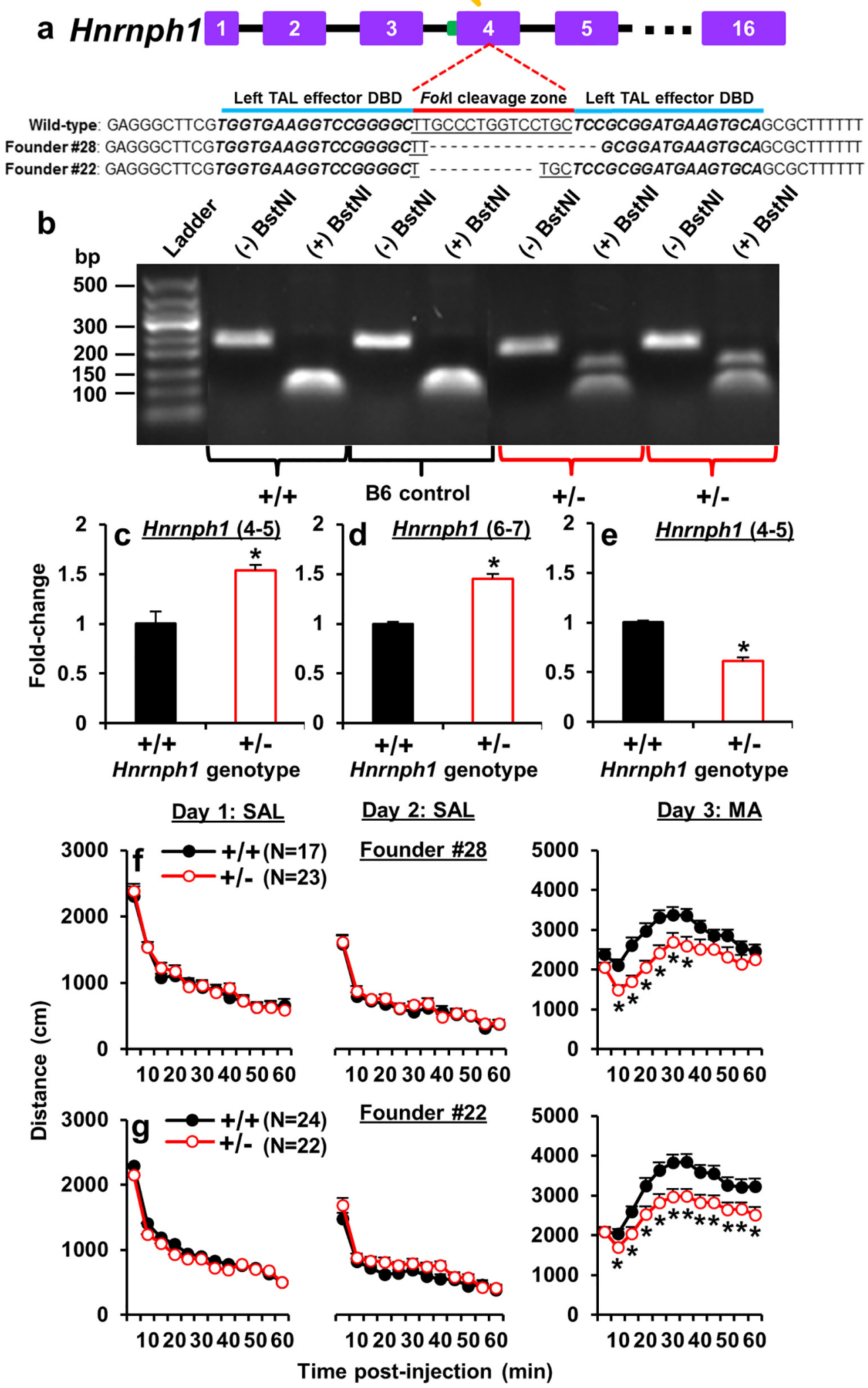


Fig 5. TALENS-targeted frameshift deletions in $H n r n p h 1^{+/-}$mice reveal Hnrnph1 as a quantitative trait gene for MA sensitivity. (a): Left TAL effector (50,191,867-50,191,883 bp) and right TAL effector $(50,191,899-50,191,915 \mathrm{bp})$ separated by the Fokl cleavage zone were used to introduce frameshift deletions in the first coding exon of Hnrnph1 (exon 4) that resulted in premature stop codons (S8 Fig). Founder \#28 contained a 16 bp deletion and Founder \#22 contained an 11 bp deletion. (b): A PCR amplicon capturing the Fokl cleavage zone was digested with BstNI. Hnrnph1 ${ }^{+/+}$mice contained two copies of a functional BstNI restriction site and thus, restriction digest produced a single band containing digested fragments of equal size. Hnrnph $1^{+/-}$mice were heterozygous for a deletion of the BstNI site and showed both the digested band and a larger, undigested band. Gel band lanes were cropped and re-ordered to present wild-type first (+/+) followed by B6 control, and heterozygous samples (+/-). (c): There was a significant upregulation of total Hnrnph1 transcript levels in Hnrnph ${ }^{+/-}$mice as indicated by cDNA amplification using qPCR primers spanning exons $4-5$ that hybridized to both genotypes $\left(t_{6}=5.69 ; p=0.0013\right)$. (d): An upregulation of total Hnrnph1 transcript levels was also indicated by cDNA amplification using qPCR primers spanning untargeted exons $6-7\left(t_{6}=8.53 ; p=0.00014\right)$. (e): A significant downregulation of the Hnrnph1 ${ }^{+/+}$ transcript levels was observed in Hnrnph $1^{+/-}$mice that was indicated by cDNA amplification using primers spanning exons $4-5$, one of which hybridized to the deleted Hnrnph $1^{+/+}$sequence $\left(t_{6}=9.45 ; p=0.00091\right.$; Fig 5e). ${ }^{*} p<0.05$. (f): In Line \#28, there was no effect of genotype on locomotor activity in response to saline (SAL) on Days 1 or 2 (left, middle panels). On Day 3, Hnrnph $1^{+-}$mice from Line \#28 heterozygotes showed a significant reduction in MA-induced locomotor activity compared to Hnrnph1 ${ }^{+/+}$littermates (right panel). (g): In Line \#22, there was no effect of genotype on locomotor activity in response to SAL on Days 1 or 2 (left, middle panels). On Day 3, Hnrnph $1^{+/-}$mice from Line \#22 showed significantly reduced MA-induced locomotor activity compared to Hnrnph $1^{+/+}$littermates. Data are presented as the mean \pm S.E.M. ${ }^{*}=$ significant genotype $x$ time interaction followed by unpaired t-tests of individual time bins $(p<0.05$; S3 Table; S1 Text; Supplementary Information).

doi:10.1371/journal.pgen.1005713.g005

for a transcription factor that is crucial for midbrain dopaminergic neuron development, survival, and cellular maintenance of the synthesis, packaging, transport, and reuptake of dopamine [36]. Nurr1 was a core component of a top-ranked gene network composed of primarily downregulated genes important for neurogenesis, neural differentiation, and synaptogenesis (Nr4a2 / Nurr1, Bdnf, Tbr1, Neurod6, Ets2, Malat1, Elavl2; Fig 4). Accordingly, there was a downregulation of striatal signaling pathways, including glutamate (Slc17a7, Slc17a6, Gng2, and Gria4), $\mathrm{G}_{\alpha \mathrm{q}}$ (Gng2, Chrm1, Adra1b, Adra1d), and GPCR signaling (Pde1b, Rgs14, Chrm1, Adra1b, Adra1d) (S8 Table). With regard to $\mathrm{G}_{\alpha \mathrm{q}}$ signaling, MA acts as a substrate for NET, causing efflux of NE [9] which then binds to $\alpha$-adrenergic receptors that are coded by Adra1b and Adra1d. Notably, knockout mice for either of these receptors exhibit reduced amphetamine-induced locomotor activity $[37,38]$.

Some of the differentially expressed genes in Line 4 a were previously associated with variation in amphetamine reward and reinforcement, including Nr4a2 (Nurr1), Adora2a, and Slc17a7 (Vglut1) [39]. Furthermore, the top predicted upstream regulator- $H t t$ (huntingtin; S7A Fig) is a master regulator of a network of genes in the extended amygdala associated with protracted abstinence from chronic exposure to opioids, cannabinoids, nicotine, and alcohol [40].

Inheritance of the Hnrnph1 locus caused downregulation of a smaller reverse-transcribed gene located within the middle of Oprm1 (mu opioid receptor) called Ipcef1 ( $\mathrm{p}=0.001$; $\mathrm{FDR}=12 \%$; S6 Table). We also identified a trans-eQTL in Hnrnph1 that regulates Ipcef1 expression (Table 2 [30]). Hnrnph1 was previously shown to regulate the expression Oprm1 (mu opioid receptor gene) via 5' UTR-mediated repression [41] and splicing [42]. Furthermore, the human intronic SNP rs9479757 in OPRM1 was associated with heroin addiction severity and decreased binding affinity of $H N R N P H 1$, resulting in exon 2 skipping [43]. Thus, Hnrnph1 regulation of Ipcef1 expression could represent an additional mechanism of Oprm1 regulation [44].

The QTL that contains Hnrnph1 is predicted to perturb the neural development of the mesocorticolimbic circuitry that mediates MA behavior. Hnrnph1 (heterogeneous nuclear ribonucleoprotein) codes for an RNA binding protein (RBP) that is highly expressed 
a

\section{Rufy1}

Left TAL effector DBD
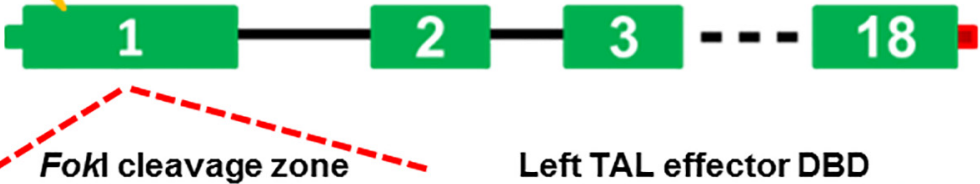

WT Rufy1: CGTGACCTAACAACATGGCCGCCCGCGGAGAATGCCGCCGGGCGGGGCAGGATT Founder \#3: CGTGACCTAACAACATGGCEGCCCGC $\ldots \ldots$
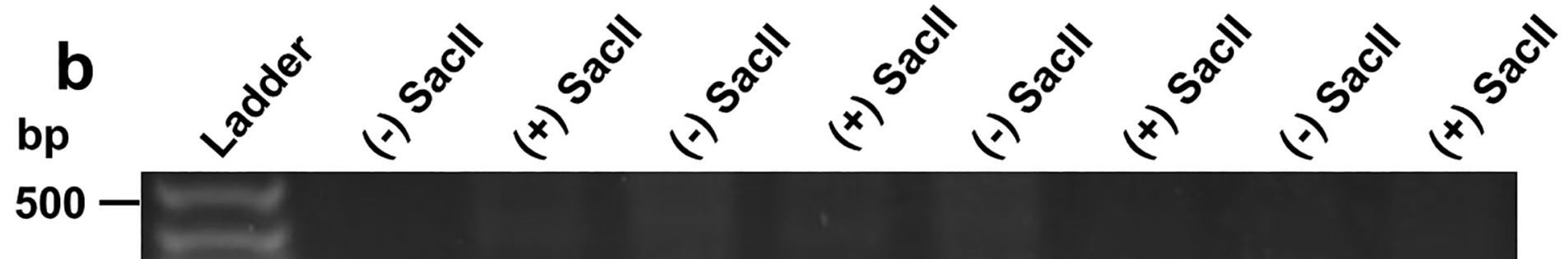

$300-\longrightarrow$

$200-$

150

$100-$
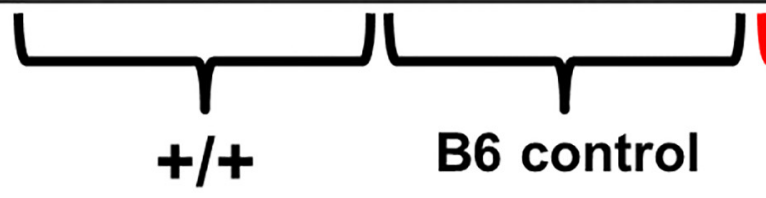

B6 control
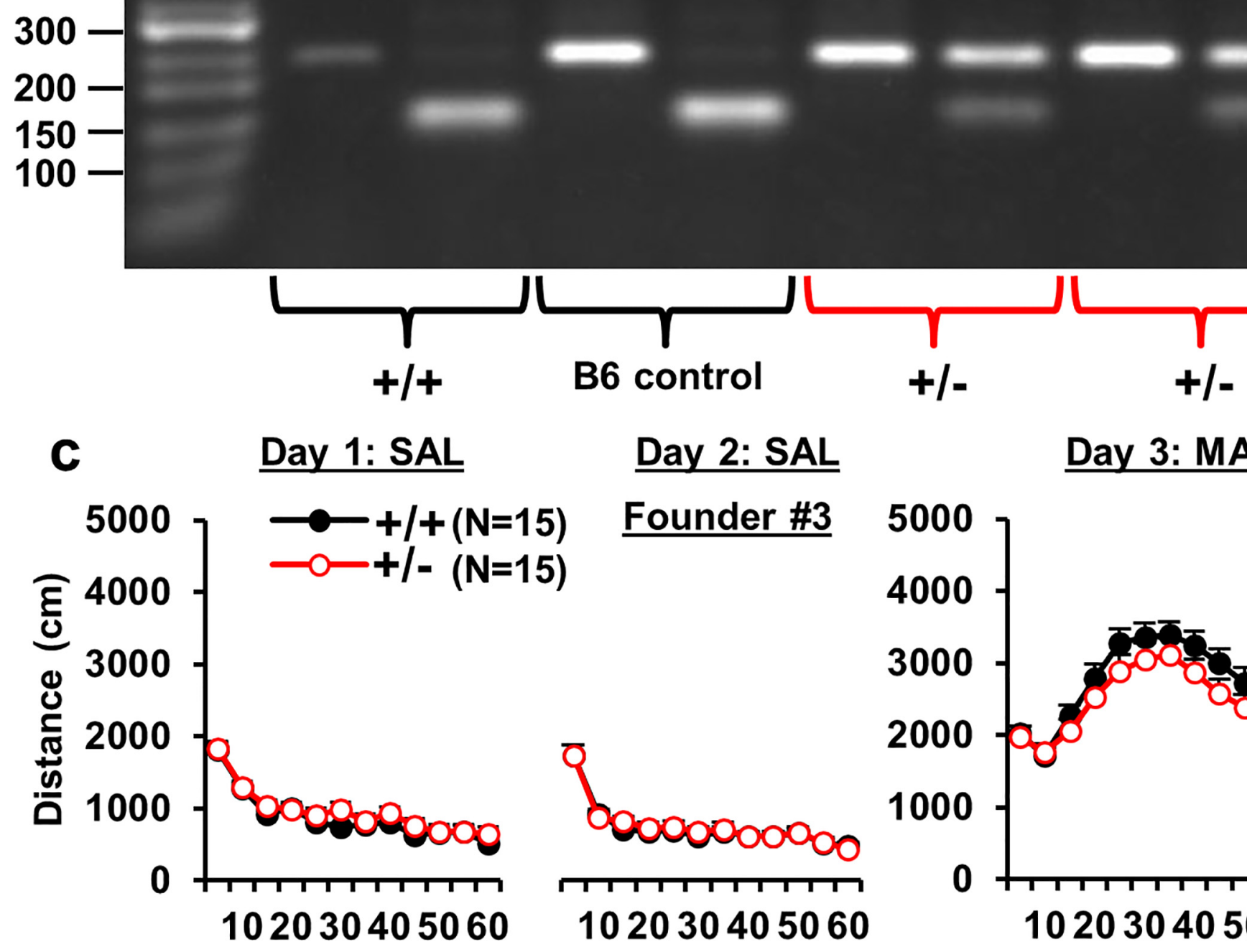

Day 2: SAL

Day 3: MA
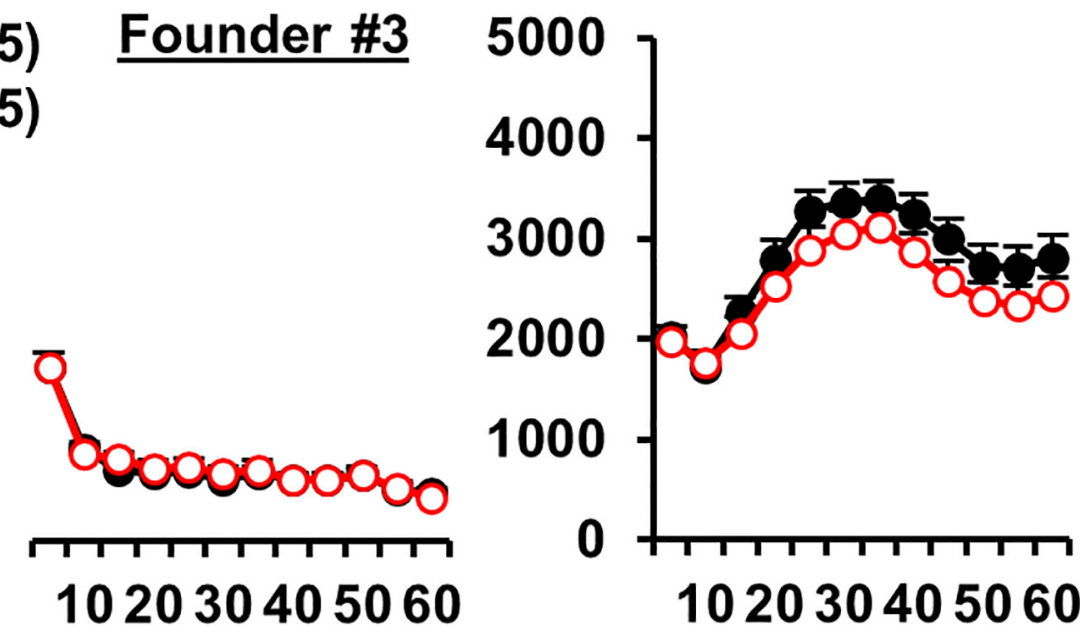

102030405060

\section{Time post-injection}

Fig 6. TALENS-targeted frameshift deletion in Rufy $1^{+/-}$mice. (a): A left TALE effector $(50,244,600-50,244,616$ bp) and a right TALE effector $(50,244,569-50,244,585 \mathrm{bp})$ separated by the Fokl cleavage zone were used to introduce a frameshift deletion that resulted in a premature stop codon in the 
first coding exon of Rufy1 (see S8 Fig). (b): A PCR amplicon was generated that captured the Fokl cleavage zone and a single Sacll restriction site and was subjected to restriction digest with Sacll. Rufy $1^{+/+}$mice contained the Sacll restriction site and thus, showed only a single, smaller band. Rufy $1^{+/-}$mice showed both the Sacll-digested band and a larger, undigested band, indicating the presence of the deletion. (c): There was no effect of genotype or genotype $x$ time interaction in Rufy $1^{+-}$versus Rufy $1^{++}$mice from Line \#3 on Days 1, 2, or 3 ( $p>0.05$; S3 Table). Data are presented as the mean \pm S.E.M.

doi:10.1371/journal.pgen.1005713.g006

throughout the brain, including the striatum, cortex, and hippocampus (S11 Fig) [34] and binds to G-rich elements to either enhance or silence splicing $[45,46]$. hnRNPs such as Hnrnph1 form hnRNP-RNA complexes to coordinate splicing of thousands of genes [46]. In addition, HNRNPH1 regulates 3' UTR cleavage and polyadenylation [47] and several hnRNPs export mRNAs to neuronal processes to regulate spatiotemporal translation and post-translational modifications [48]. Synaptic activity can increase protein abundance of hnRNPs at the post-synaptic density of primary neurons [49]. The hippocampus contains focal expression of over 15 hnRNPs, including H1 (S11 Fig [34]). Importantly, Hnrnph1 contains a glycine rich domain that permits nucleocytoplasmic shuttling via transportin 1 [50] and exhibits activitydependent translocation to the cytoplasm [51]. Several hnRNPs exhibit activity-dependent localization at the synapse [49], suggesting additional neuronal functions of Hnrnph1 in addition to splicing.

We identified Hnrnph1 as a quantitative trait gene responsible for MA sensitivity. However, the quantitative trait nucleotide(s) remain obscure. Hnrnph1 contains 18 genetic variants within the gene, including 15 intronic SNPs, a SNP in the 5' UTR, a synonymous coding SNP, and a single T insertion in the 3' UTR (S4 Table $[52,53]$ ) that could cause brain region-specific differential expression of Hnrnph1 and/or its ability to regulate splicing of its transcriptomewide targets [46, 47]. We did not observe differential striatal expression of Hnrnph1 at the gene level or the exon level as a consequence of inheriting the Line 4a QTL (S5 and S6 Figs). Our focus was limited to the striatum which is a behaviorally relevant region $[16,29]$ that exhibits high Hnrnph1 expression during early adulthood (S11 Fig). Therefore, the QTL could influence Hnrnph1 expression at a different time period, in a different, behaviorally relevant brain region, or in a specific subpopulation of cells. Interestingly, striatal microarray datasets in BXD strains indicate an increase in Hnrnph1 expression from postnatal day 3 to postnatal day 14 as well as a change in the strain rank order of expression [30] which suggests that genotypic differences in Hnrnph1 expression could depend on the developmental time point. Finally, because excised introns can trans-regulate gene expression, an alternative explanation is that excised, SNP-containing introns from Hnrnphl can function as polymorphic long noncoding RNAs to perturb their trans-regulation of the transcriptome [54].

To our knowledge, there are no GWAS studies reporting genome-wide significant associations of HNRNPH1 variants with complex diseases or traits (http://www.ebi.ac.uk/gwas/). Interestingly, HNRNPH1 binding affinity and splicing can be modulated by genome-wide significant SNPs associated with bipolar disorder, major depressive disorder, and schizophrenia, including rs1006737 (CACNA1C), rs2251219 (PBRM1), and rs1076560 (DRD2) [55]. Thus, $H N R N P H 1$ splicing could profoundly impact the neurobiological mechanisms underlying these disorders. Additionally, HNRNPH1 and RBFOX1/2 coordinate splicing [56, 57] and knockdown RBFOX1 (an autism-associated RBP involved in neural development [58]) in human neural progenitor cells revealed over 200 alternatively spliced genes containing HNRNPH1 binding sites [56] and 524 genes containing binding sites for ELAVL2, a neurodevelopmental RBP [59] that was downregulated in Line 4a (Fig 4).

In summary, we identified Hnrnph1 as a quantitative trait gene for MA sensitivity. This is rarely accomplished in rodent forward genetic studies of behavior and will likely advance our understanding of the neurobiological basis of multiple neuropsychiatric disorders involving 
monoaminergic dysregulation. Identifying brain region- and cell type-specific splicing targets of Hnrnph 1 could reveal therapeutic targets for these disorders, many of which have been associated with specific gene splicing events [55]. Furthermore, pharmacological perturbation of RBP function could one day serve as an effective therapeutic strategy. Recent findings in models of neurodegenerative disease show that targeting RBP signaling could be a promising treatment approach [60].

\section{Materials and Methods}

\section{Mice}

All procedures in mice were approved by the Boston University and the University of Chicago Institutional Animal Care and Use Committees and were conducted in strict accordance with National Institute of Health guidelines for the care and use of laboratory animals. Colony rooms were maintained on a 12:12 h light-dark cycle (lights on at $0600 \mathrm{~h}$ ). Mice were housed in same-sex groups of two to five mice per cage with standard laboratory chow and water available ad libitum. Age-matched mice were 50-100 days old at the time of testing (0900-1600 h).

\section{Locomotor activity}

For Lines 1-6 and Lines 4a-4h, locomotor activity was assessed in the open field [19]. Briefly, congenics, subcongenics, and wild-type littermates were transported from the vivarium to the adjacent behavioral testing room where they habituated for at least $30 \mathrm{~min}$ prior to testing. Mice were then placed into clean holding cages with fresh bedding for approximately five min before receiving an injection of saline on Days 1 and $2(10 \mu \mathrm{l} / \mathrm{g}$, i.p) and an injection of methamphetamine on Day 3 (MA; 2 mg/kg, i.p.; Sigma-Aldrich, St. Louis, MO USA). Mice were placed into the center of the open field $(37.5 \mathrm{~cm}$ x $37.5 \mathrm{~cm}$ x $35.7 \mathrm{~cm}$; AccuScan Instruments, Columbus, OH USA) surrounded by a sound attenuating chamber (MedAssociates, St. Albans, VT USA) and the total distance traveled was recorded in six, 5 min bins over 30 min using VersaMax software (AccuScan).

Mice heterozygous for a frameshift deletion in Hnrnph1 (Hnrnph1 $\left.{ }^{+/-}\right)$or Rufyl $\left(R u f y 1^{+/-}\right)$ were engineered (http://www.bumc.bu.edu/transgenic/), bred, and phenotyped at Boston University School of Medicine. Mice were bred and phenotyped in a manner similar to the congenics at the University of Chicago, with the exception that the open field was a smaller size (43.2 cm long x $21.6 \mathrm{~cm}$ wide $x 43.2 \mathrm{~cm}$ tall; Lafayette Instruments, Lafayette, IN USA) and mice were recorded daily for $1 \mathrm{~h}$ rather than $30 \mathrm{~min}$ to allow a more robust detection of the phenotype. Reduced MA sensitivity was also replicated in Hnrnph $1^{+/-}$mice using the $30 \mathrm{~min}$ protocol (Supplementary Information). Behavior was videotaped using a security camera system (Swann Communications, Melbourne, Australia) and data were collected and analyzed using video tracking (Anymaze, Stoelting, Wood Dale, IL USA).

\section{Behavioral analysis}

Because our primary focus was on MA-induced locomotor activity on Day 3, we first ran a two-way repeated measures ANOVA for Day 3 using genotype and sex as factors and time as the repeated measure. Because sex did not interact with genotype or time for any of the lines on Day 3, we combined sexes for the analysis of Days 1-3 and used repeated measures ANOVA with genotype as the main factor. Main effects of genotype and genotype $\mathrm{x}$ time interactions were deconstructed using one-way ANOVAs and Fisher's post-hoc test of each time bin or t-tests in cases where there were two genotypes. A p-value of less than 0.05 was considered significant. 


\section{QTL analysis of $\mathrm{F}_{2}$ mice}

$\mathrm{B} 6 \times \mathrm{D} 2-\mathrm{F}_{2}$ mice $(\mathrm{N}=676)$ were generated, maintained, genotyped, and analyzed as previously described [20, 22]. Genome-wide QTL analysis was performed in $\mathrm{F}_{2}$ mice using the R package QTLRel that contains a mixed model to account for relatedness among individuals [61]. We recently validated the use of permutation when estimating significance thresholds for mixed models [62]. Sex was included as an interactive covariate. For each analysis, significance thresholds ( $\mathrm{p}<0.05$ ) were estimated using 1000 permutations. The $\mathrm{F}_{2}$ data and $\mathrm{R}$ code for are publicly available on github (https:/github.com/wevanjohnson/hnrnph1).

\section{Generation of congenics and subcongenics}

Lines 1 and 6 were obtained from Dr. Aldons Lusis's laboratory at UCLA (Lines "11P” and "11M" [28]) and had previously been backcrossed to B6 for more than 10 generations. These lines contained homozygous, introgressed regions from D2 on an isogenic B6 background that spanned chromosome 11. Because Lines 1 and 6 contained such large congenic intervals, we first phenotyped non-littermate offspring derived from homozygous congenic breeders versus homozygous B6 wild-type breeders (The Jackson Laboratory, Bar Harbor, ME; Figs 2 and S2) rather than heterozygous-heterozygous breeders to avoid the otherwise high likelihood of introducing unmonitored recombination events. Thus, we ensured that each individual possessed an identical genotype within each congenic line. The same type of control group is typically employed in the initial screen of chromosome substitution strains $[19,63,64]$ which are essentially very large congenic lines. We crossed Line 1 to $\mathrm{B} 6$ and phenotyped the $\mathrm{F}_{1}$ offspring alongside age-matched B6 mice. B6 cohorts were combined into a single group for the combined analysis of all three genotypes for Line 1 (homozygous for B6, homozygous for D2, and heterozygous; Fig 2).

Next, we backcrossed Line 1 heterozygotes to B6 to generate subcongenic Lines 2-5 (Figs 2 and S2). Recombination events were monitored using genomic DNA extracted from tail biopsies and a series of TaqMan SNP markers (Life Technologies; Carlsbad, CA; S1 Table). We then used heterozygous-heterozygous breeding in Lines 2-5 to produce littermates of all three genotypes for simultaneous phenotyping (Figs 2 and S2). Because the QTL in Line 4 represented the smallest congenic region and was dominantly inherited, we backcrossed Line 4 heterozygotes to B6 to generate heterozygotes and wild-type littermates for Lines 4a-4h (Figs 3 and S3). We used additional TaqManSNP markers (Life Technologies) to monitor recombination events and defined the precise congenic boundaries using PCR and Sanger sequencing of SNPs chosen from the Mouse Sanger SNP query database (http://www.sanger.ac.uk/cgi-bin/ modelorgs/mousegenomes/snps.pl [52]). Genomic coordinates are based on mm9 (Build 37).

\section{Test for residual heterozygosity in Lines 4a, 4b, 4c, and 4d}

We assayed tail SNP DNA from one heterozygous congenic mouse and one B6 wildtype littermate from Lines $4 \mathrm{a}-4 \mathrm{~d}$ (eight mice total) using services provided by the DartMouseSpeed Congenic Core Facility at the Geisel School of Medicine at Dartmouth College (http://dartmouse. org/). A total of 882 informative B6/D2 SNPs were analyzed on the GoldenGate Genotyping Assay (Illumina, Inc., San Diego, CA) using DartMouse's SNaP-Mapand Map-Synth software to determine the allele at each SNP location. After detecting a single off-target locus on chromosome 3 (rs13477019; $23.7 \mathrm{Mb}$ ), we used a custom designed TaqMan SNP marker for rs13477019 (Life Technologies, Carlsbad, CA USA) to confirm the result and to genotype additional samples from Lines $4 \mathrm{a}-4 \mathrm{~h}$ for which we had both DNA and behavioral phenotypes. Data from this SNP marker were then used to test for the effect of genotype at the chromosome 3 locus on MA-induced locomotor activity. 


\section{RNA-seq}

We harvested and pooled bilateral $2.5 \mathrm{~mm}$ diameter punches of the striatum for each individual sample from naïve, congenic mice and B6 wildtype littermates from Line 4a ( $\mathrm{N}=3$ females and 5 males per genotype; 50-70 days old). Total RNA was extracted as previously described [23] and purified using the RNeasy kit (Qiagen, Valencia, CA, USA). RNA was shipped to the University of Chicago Genomics Core Facility where cDNA libraries were prepared for $50 \mathrm{bp}$ single-end reads according to the manufacturer's instructions using the Illumina TruSeqStranded mRNA LT Kit (Part\# RS-122-2101). Purified DNA was captured on an Illumina flow cell for cluster generation and sample libraries were sequenced at eight samples per lane over two lanes (technical replicates) on the Illumina HiSeq 2500 machine according to the manufacturer's protocols. FASTQ files were quality checked via FASTQC and possessed Phred quality scores $>30$ (i.e. less than $0.1 \%$ sequencing error). Using the FastX-Trimmer from the FastX-Toolkit, the 51st base was trimmed to enhance read quality and prevent misalignment. FASTQ files were utilized in TopHat [65] to align reads to the reference genome (UCSC Genome Browser). Read counts per gene were quantified using the HTSeq Python package and the R Bioconductor package edgeR was used to analyze differential gene expression. EdgeR models read counts using a negative binomial distribution to account for variability in the number of reads via generalized linear models [66]. "Home cage" was included as a covariate in the statistical model to account for cage effects on gene expression. The p-values obtained for differential expression were then adjusted by applying a false discovery rate (FDR) method to correct for multiple hypothesis testing [67]. The transcriptome dataset and code for RNAseq analysis are available via NCBI Gene Expression Omnibus (http://www.ncbi.nlm.nih.gov/ geo/query/acc.cgi?token=cxkdoeaudvyhlqt\&acc=GSE66366).

\section{Real-time quantitative PCR (qPCR)}

Oligo-dT primers were used to synthesize cDNA from total RNA to examine mRNA expression. Primer efficiencies for real-time quantitative PCR (qPCR) experiments were calculated

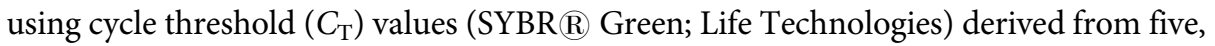
10 -fold serial cDNA dilutions; efficiencies (E) ranged from $90-100 \%\left(\mathrm{R}^{2}=0.99-1\right)$. Each sample was run in triplicate and averaged. Differential gene expression was reported as the foldchange in congenic or frameshift-deleted mice relative to B6 wild-type littermates using the $2^{-\left(\Delta \Delta \mathrm{C}_{\mathrm{T}}\right)}$ method [68].

\section{Ingenuity pathway analysis (IPA)}

We used our differentially expressed gene list from the striatal transcriptome that contained both the $\log _{2}$ fold-change and p-values (FDR < 5\%) and applied IPA (www.qiagen.com/ ingenuity) to identify enriched molecular pathways, functional annotations, gene networks, upstream causes, and predicted neurobiological consequences caused by inheritance of the QTL. IPA utilizes an algorithm that assumes that an increase in the number of molecular interactions indicates an increase in the likelihood of an effect on biological function. IPA uses a manually curated database (IPA Knowledge Base) containing the published literature to extract gene networks containing equally treated edges that directly and indirectly connect biologically related genes (www.qiagen.com/ingenuity). IPA analyses were conducted in February 2015.

IPA settings. We considered both direct and indirect relationships that were experimentally observed or moderately-to-highly predicted in all mammalian species, including mouse and rat. We used the "stringent" setting to filter molecules and relationships in tissues and cell lines. With regard to mutations, we considered all functional effects, modes of inheritance, translational impacts, zygosity, wild-type, and unclassified mutation information. 
Canonical pathways. The ratio of the canonical pathways represents the number of genes in our gene list that overlap with the genes listed in the IPA-generated pathway divided by the total genes within the IPA-generated pathway; thus, a ratio equal to 1 represents perfect overlap. The- $\log _{10}$ (p-value) for each canonical pathway was derived from the right-tailed Fisher's exact that measured the degree of overlap between the number of genes identified in our list with the number of genes that comprise the canonical pathway versus the number of genes genome-wide that would be expected to overlap by chance. The p-values were corrected for multiple testing using the Benjamini-Hochberg method [67] and represent the FDR.

Diseases, functions, and gene networks. The statistical significance of overlap between our gene list and a particular disease or function was assessed using the p-value derived from a Fisher's exact test. The predicted activation state was assessed by calculating a z-score that determined the statistical significance of the match between the observed and predicted direction. "Increased" or "decreased" indicates that the Z-score was significant for predicting activated or inhibited state. IPA networks were built based on the degree of connectivity between genes within our gene list, starting with the most connected genes. Genes were added by the IPA algorithm to the network to facilitate connectivity. Networks were limited to a maximum of 35 genes to facilitate interpretability and the ability to generate hypotheses. The Network Score (see S10 Table), a.k.a., the "p score", represents the- $\log _{10}$ (p-value) and represents the probability of finding the observed number of focus genes in a network by chance.

Upstream regulator analysis. This analysis identifies causal molecules associated with differential expression using both the significance and the direction of differential expression to specify causal predictions. Several plausible causal networks are constructed and used to calculate an enrichment score and p-value based on overlap between predicted and observed regulator-regulated genes (Fishers exact test). A Z-score is also calculated that determines the degree of match between observed and predicted direction of gene expression (+ or-[69]).

"Increased" or "decreased" indicates that the Z-score was significant for predicting activation or inhibition of the regulator.

\section{GeneNetwork}

To identify published cis- and trans- eQTLs that could explain gene expression differences caused by inheritance of the Line 4 a congenic interval, we queried differentially expressed genes (FDR $<20 \%$; 174 genes total; S6 Table) in transcriptome datasets from several brain regions in GeneNetwork [30] involving BXD recombinant inbred strains (recombinant inbred strains derived from B6 and D2 strains). We considered cis- and trans-QTLs originating from SNPs located within the 50-60 Mb locus and employed an arbitrary cut-off of LRS $\geq 13.8$ $(\mathrm{LOD} \geq 3)$. We only included genes where there was an exact match of gene with the LRS location using the appropriate genome build coordinates for each dataset.

\section{Generation of TALENs-targeted Hnrnph $1^{+/-}$and Rufy $1^{+/-}$mice}

TALENs vectors encoded either the right or left arm of the TALE effector that targeted the first coding exons of Hnrnph1 or Rufy1 (Cellectis Bioresearch, Paris, France). Upon bacterial cloning and purification, TALENs vectors containing a T7 promoter were linearized and used as templates for in vitro mRNA synthesis (mMessage mMachine T7 transcription kit; Life Technologies), and purified using MEGAclear transcription clean-up kit (Life Technologies). Each mRNA cocktail was diluted in sterile buffer and injected into B6 single-cell embryos at the BUMC Transgenic Core facility (http://www.bumc.bu.edu/transgenic/). We developed a genotyping assay utilizing native restriction enzyme recognition sites within the TALENs FokI cleavage domain. Genomic DNA was extracted from mouse tail biopsies and PCR-amplified 
with primers targeting 100 base pairs upstream and downstream of the TALENs binding domain. Amplicons were then exposed to restriction digest overnight, run on a $2 \%$ agarose Ethidium Bromide Tris-Borate-EDTA gel, and imaged with ultraviolet light. TALENs-targeted deletions were identified by the presence of undigested bands caused by a loss of the restriction site. To confirm base pair deletions in our founder lines, undigested restriction enzymeexposed PCR amplicon bands were excised, gel-purified, and vector-ligated overnight at $4^{\circ} \mathrm{C}$ using the pGEM T-easy Vector Systems (Promega). The ligation reaction was transformed into MAX Efficiency DH5 $\alpha$ Competent Cells (Invitrogen) and plated onto Ampicillin-IPTG/X-Gal $\mathrm{LB}$ agarose plates for blue-white selection. Following overnight incubation at $37^{\circ} \mathrm{C}$, white colonies were picked, cultured in ampicillin-enriched LB medium, and amplified. The PCR product was purified using the QIAprep Miniprep kit (QIAGEN). We then sequenced the vectors for the deletions using the pGEM T7 site upstream of the insert.

\section{Genotyping of TALENs-targeted Hnrnph $1^{+/-}$and Rufy $1^{+/-}$mice}

An Hnrnph1 forward primer (GTTTTCTCAGACGCGTTCCT) and reverse primer (ACTGA CAACTCCCGCCTCA) were designed to target upstream and downstream of the TALENs binding domain in exon 4 of Hnrnph1. Genomic DNA was used to amplify a 204 bp PCR product using DreamTaq Green PCR Mastermix (ThermoScientific). PCR products were treated with the BstNI restriction enzyme (New England Biolabs) or a control enzyme-free buffer solution and incubated overnight at $60^{\circ} \mathrm{C}$ to ensure complete digestion. Enzyme-treated PCR products and untreated controls were resolved in $2 \%$ agarose gel electrophoresis with $0.5 \mu \mathrm{g} / \mathrm{mL}$ ethidium bromide to visualize under UV light. There were two BstNI restriction sites within the Hnrnph1 amplicon that were located proximal and distal to the TALENs FokI cleavage zone. Mice heterozygous for the Hnrnph1 deletion showed two bands on the gel, while B6 controls showed a single band.

Similar to Hnrnph1, a Rufyl forward primer (AATCGTACTTTCCCGAATGC) and reverse primer (GGACTCTAGGCCTGCTTGG) targeted upstream and downstream of the TALENs binding domain in the first coding exon (exon 1). The $230 \mathrm{bp}$ PCR amplicon contained a SacII restriction site that was deleted in Rufy $1^{+/-}$mice. Thus, Rufy $1^{+/+}$mice showed a single, smaller digested band whereas $R u f y 1^{+/}$mice showed both the digested band as well as a larger, undigested band.

\section{Assessment of potential off-target deletion of Hnrnph2 in Hnrnph1- targeted mice}

To assess off-target activity in Hnrnph1-targeted mice, we used the UCSC genome browser to BLAT the TALENs binding domains and identified a single homologous region located within the first coding exon of Hnrnph2. We used the same PCR- and gel-based assay to test for the deletion in Hnrnph2 with the exception that we used forward (GCCACCAAGAGTCCATCAGT) and reverse primers (AATGCTTCACCACTCGGTCT) that uniquely amplified a homologous 197 bp sequence within Hnrnph2 that contained a single Bstn1 restriction site. Digestion at the Bstn1 site produced an 81 bp band and a 115 bp band.

\section{Supporting Information}

S1 Text. Statistical analyses are provided for Lines 1-6 and Lines 4a-4h. Additional details are also provided for assessment of residual heterozygosity and statistical analyses for Hnrnph $1^{+/-}$and Rufy $1^{+/-}$mice.

(DOCX) 
S1 Table. SNPs that define Lines 1 through 6. B6 = homozygous for C57BL/6); $\mathrm{D} 2$ = homozygous for DBA/2J; $\mathrm{H}$ = heterozygous; ARRAY = SNP array-based genotyping; TAQMAN = custom-designed fluorescent SNP genotping; SEQ = Sanger sequencing-based genotyping; $\mathrm{ND}=$ not determined (XLSX)

S2 Table. SNPs that define Lines 4a-4h. SNP ID, chromosome 11 location (mm9) method of genotyping, and genotypes are listed. B6 = homozygous for C57BL/6); D2 = homozygous for $\mathrm{DBA} / 2 \mathrm{~J} ; \mathrm{H}=$ heterozygous; ARRAY = SNP array-based genotyping; SEQ/TAQ = SNPs were both Sanger-sequenced and genotyped using custom-designed Taqman fluorescent SNP genotyping; $\mathrm{SEQ}=$ Sanger sequencing-based genotyping; $\mathrm{NI}=$ non-informative; $\mathrm{ND}=$ not determined. Red-filled cells denote the critical interval spanning 50,185,512-50,391,845 bp. (XLSX)

S3 Table. ANOVA tables for congenic lines and TALENs-targeted lines. F statistics and pvalues are listed for Days 1, 2, and 3 for the effect of genotype (Geno) and Geno $\mathrm{x}$ Time interactions as well as significant time bins.

(XLSX)

S4 Table. Genetic variants between B6 and D2 within critical interval. Data ( $\mathrm{mm} 9)$ were obtained from the Sanger mouse query tool containing genetic variants (http://www.sanger.ac. $\mathrm{uk} /$ resources/mouse/genomes/). (XLSX)

S5 Table. Residual heterozygosity in Lines 4a through 4d. One mouse for each genotype (B6 = homozygous for B6 allele; $\mathrm{H}=$ heterozygous for B6 and D2 alleles) from each of the four congenic lines (Lines $4 \mathrm{a}, 4 \mathrm{~b}, 4 \mathrm{c}$, and $4 \mathrm{~d}$ ) were genotyped using services provided by DartMouse. The SNP ID, chromosome (Chr.), physical position (Build 34), and genotype are listed. Each SNP allele is represented by either "A" or "B" and the reference allele ("JAX B6") which could be either AA or BB. $\mathrm{NC}=$ no call.

(XLSX)

S6 Table. Differentially expressed genes in the striatum of Line 4a. Gene ID, gene name, physical position and build, log2 fold-change, fold-change (FC), P-value, and Q-value are listed in order of ascending $\mathrm{p}$-value.

(XLSX)

S7 Table. Primer sequences used for qPCR. Genes, targeted exons, forward and reverse sequences, and amplicon size (bp) are listed.

(XLSX)

S8 Table. Canonical pathways in IPA. The pathway, -logP, ratio, z-score, and genes ("Molecules") identified from our list are shown. The top 20 annotations are listed. (XLSX)

S9 Table. Diseases and functions annotations. The z-score indicates the degree of match between the observed and predicted "Increased" or "decreased" denotes those Z-score that were significant.disease or function. The top 20 annotations are shown.

(XLSX)

S10 Table. Top IPA networks containing disease and functions annotations. Score [p score; - $\log 10$ (p-value], number of focus genes identified from our gene list, and names of diseases 
and function associated with each network are shown.

(XLSX)

S1 Fig. Distal QTL on chromosome $11(90 \mathrm{Mb})$ for days 1 and 2 that increased locomotor activity in response to saline $(\mathrm{D2}>\mathrm{B6})$. We previously published a genome-wide significant QTL on chromosome 11 for Day 1 and Day 2 from this B6 $\times$ D2- $\mathrm{F}_{2}$ dataset that was significant from $0-15 \mathrm{~min}$ and from $15-30 \mathrm{~min}^{20}$. Here, we report the LOD scores from the same dataset in six, 5-min time bins over $30 \mathrm{~min}$. (a, b): QTL plots are shown for the time bins on Day 1 (saline; SAL, i.p.) and Day 2 (SAL, i.p.). The $\mathrm{x}$-axis represents the physical location of the marker $(\mathrm{Mb})$. The $\mathrm{y}$-axis represents the LOD score. The dashed, horizontal line represents the genome-wide significance threshold derived from 1,000 permutations. The dashed QTL trace indicates the time bin containing the most significant LOD score for each day. The peak LOD was observed at approximately $90 \mathrm{Mb}$; this same QTL was also present on Day 3 at the first 5-min bin prior to the behavioral onset of MA (Fig 1A). (c, d): Effect plot of the marker with the most significant LOD scores is shown for Day 1 and Day 2 in 5-min time bins. Data are sorted by genotype at the marker rs3710148 (96.4 Mb) for each time bin. The time bin with the most significant LOD score is circled. B6 = homozygous for the B6 allele (black circles); $\mathrm{H}=$ heterozygous (open triangles); D2 = homozygous for the D2 allele (colored squares). Data are presented as the mean \pm S.E.M.

(TIF)

S2 Fig. MA sensitivity in Line 5 and Line 6. Lines 5 and 6 possessed chromosome 11 intervals from the D2 strain on an isogenic B6 background (see Fig 2A). The SNPs used to define the intervals in Lines 5 and 6 are listed in S1 Table. (a, b): The three columns represent the locomotor phenotypes for Days 1, 2, and 3 for Line 5 and Line 6. Sample sizes (N) are listed for each genotype. Data are presented as the mean \pm S.E.M. Statistical analyses are included in S3 Table. (TIF)

S3 Fig. MA sensitivity in Lines 4d-4h. Lines $4 d-4 h$ were derived from Line 4 and possessed heterozygous intervals from the D2 strain on an isogenic B6 background (see Fig 3A). The SNPs used to define Lines 4d-h are listed in S2 Table. (a-e): The three columns represent the locomotor phenotypes for Days 1,2, and 3. The five rows (a-e) represent the phenotypes for Lines $4 \mathrm{~d}-4 \mathrm{~h}$, respectively. Sample sizes (N) are listed for each genotype. There was no effect of genotype or genotype $\mathrm{x}$ time interaction on MA-induced locomotor activity for any of these lines (see S3 Table). Data are presented as the mean \pm S.E.M. (TIF)

S4 Fig. Physical map of the 882 genome-wide informative markers used to ascertain residual heterozygosity in Lines 4a-4d. (a): The sample that is shown is a Line 4a heterozygous mouse that was genotyped with the GoldenGate SNP microarray (services and figure were provided by DartMouse; http://dartmouse.org/). As expected, this mouse was heterozygous for B6 and D2 alleles at all three SNP markers within the Line 4a congenic region on chromosome 11 (purple, horizontal ticks). Additionally, this mouse was heterozygous at a marker located on chromosome 3 (rs13477019; $23.7 \mathrm{Mb}$; purple, horizontal tick). This region of residual heterozygosity also segregated in Lines $4 \mathrm{~b}-4 \mathrm{~h}$. All other markers were genotyped as homozygous for the B6 allele (green, horizontal ticks). S5 Table lists the complete set of SNPs and genotypes for the eight samples tested on the array. (b): When sorting by genotype on chromosome 3 (rs13477019) in 115 mice from Lines 4a-4h for which we had both genotypic and phenotypic information available, there was no effect of genotype $\left(\mathrm{F}_{2,112}<1\right)$ or genotype $\mathrm{x}$ time interaction with regard to MA sensitivity $\left(\mathrm{F}_{5,560}<1\right)$. Data are presented as the mean \pm S.E.M. (TIF) 
S5 Fig. qPCR results for Hnrnph1 and Rufy1 expression in the striatum in Line 4a. (a): Heterozygous $(\mathrm{H})$ mice $(\mathrm{N}=8)$ showed significantly reduced Nurrl expression relative to B6 $\left(\mathrm{N}=8 ; \mathrm{t}_{14}=2.18 ; \mathrm{p}=0.047\right)$. $(\mathrm{b}, \mathrm{c})$ : There was no significant difference in expression of Hnrnph1 (exons 12-13; $\mathrm{t}_{29}<1$ ) or Rufy1 (exons $16-17 ; \mathrm{t}_{29}=1.51 ; \mathrm{p}=0.14$ ) in $\mathrm{B} 6(\mathrm{~N}=14$ ) versus $\mathrm{H}(\mathrm{N}=17)$ mice. Data are presented as the mean \pm S.E.M. Primer sequences are listed in $\mathrm{S} 7$ Table.

S6 Fig. Exon-level read counts for Hnrnph1 and Rufy1 in Line 4a using Integrated Genome Browser. (a, b): The $\mathrm{x}$-axis represents the physical location $(\mathrm{bp})$ of the annotated exons (vertical lines, UCSC Genome Browser; mm9) on chromosome 11 for Hnrnph1 and Rufyl. The yaxis represents the summed read counts (y-axis) across all 8 samples for each genotype (B6, $\mathrm{H}$ ). Note that different scales are used on the y-axis for Hnrnph1 (the more highly expressed gene; $0-2500$ reads) versus Rufy1 (0-300 reads).

S7 Fig. $\mathrm{Htt}$ and $\mathrm{Creb} \mathbf{1}$ are the top two IPA upstream regulators of the striatal transcriptome in Line 4a. Arrows pointing toward genes indicate predicted activation; horizontal, perpendicular lines indicate predicted inhibition. Green and red colors indicate downregulated or upregulated genes in our dataset. Purple circles denote genes that overlap between $\mathrm{Htt}$ (a) and Creb1 (b). The legend on the right hand side denotes the biological classification for each gene contained in the regulator diagrams.

(TIF)

S8 Fig. TALENs-targeted Hnrnph1 and Rufy1 deletions produce frameshift mutations that result in premature stop codons. We used the ExPASy Translate Tool (http://web.expasy.org/ translate/) to input wild-type and deleted cDNA sequences to obtain protein sequences. (a-c): Amino acid sequence is shown for Hnrnph $1^{+/+}$mice and Hnrnph $1^{+/-}$founders. (d, e): Amino acid sequence is shown for Rufy $1^{+/+}$and $R u f y 1^{+/-}$founders. Methionine (Met) is shown in green. A red "Stop" denotes a stop codon.

S9 Fig. No off-target deletions in the highly homologous Hnrnph2 gene and no compensatory change in Hnrnph2 expression in Hnrnph $\mathbf{1}^{+/-}$mice. (a): A 197 bp PCR amplicon was generated using primers specific for exon 4 of Hnrnph 2 and contained the same homologous BstNI cut site as exon 4 in Hnrnph1 (Fig 5). Hnrnph1 ${ }^{+/+}$mice and Hnrnph1 ${ }^{+/}$founder mice (\#28 and \#22) that were heterozygous for an Hnrnph1 frameshift deletion all showed two bands following restriction digest, indicating that there was no deletion of the restriction site in Hnrnph2. (b): There was no compensatory change in Hnrnph2 expression in Line \#28 when comparing Hnrnph1 $1^{+/-}(\mathrm{N}=4)$ versus Hnrnph1 ${ }^{+/+}(\mathrm{N}=4)$ mice $\left(\mathrm{t}_{6}<1\right)$. Data are presented as the mean \pm S.E.M.

S10 Fig. Reduced MA sensitivity in TALENs-targeted Hnrnph ${ }^{+/-}$mice (Founder \#28 Line) following 30 min training sessions. (a): For Day 1 , there was no effect of genotype $\left(\mathrm{F}_{1,32}<1\right)$ nor any interaction with time $\left(\mathrm{F}_{5,160}<1\right)$. (b): For Day 2, there was no effect of genotype $\left(\mathrm{F}_{1,32}=3.79 ; \mathrm{p}=0.06\right)$ but there was a significant genotype $\mathrm{x}$ time interaction $\left(\mathrm{F}_{5,160}=3.66\right.$; $\mathrm{p}=0.0037$ that was explained by Hnrnph $1^{+/-}$mice showing significantly greater locomotor activity than Hnrnph $1^{+/+}$mice at the 5-min and 10-min time bins $\left(\mathrm{t}_{32}=2.53,2.42 ; \mathrm{p}=0.017\right.$, 0.021). (c): For Day 3, there was an effect of genotype $\left(F_{1,32}=5.37 ; p=0.027\right)$ but no significant genotype $\mathrm{x}$ time interaction $\left(\mathrm{F}_{5,160}=2.04 ; \mathrm{p}=0.076\right)$. Hnrnph1 ${ }^{+/}$mice showed significantly 
less MA-induced locomotor activity than Hnrnph $1^{+/+}$mice at 25 and $30 \mathrm{~min}\left(\mathrm{t}_{32}=2.07,3.03\right.$; $\mathrm{p}=0.046,0.0048)$. Data are presented as the mean \pm S.E.M. ${ }^{*} \mathrm{p}<0.05$.

(TIF)

S11 Fig. Mid-sagittal, in situ hybridization sections for Hnrnph1 and Rufy1. In situ hybridization staining of mid-sagittal sections are shown for Hnrnph1 (panel a) and Rufyl (panel b) and were obtained from the Allen Institute for Brain Science (http://www.brain-map.org/ ${ }^{4}$ ). Hnrnph1 clearly shows higher expression than Rufy1 which can also evident in the number of read counts in our dataset (see also S6 Fig).

(TIF)

\section{Acknowledgments}

We would like to acknowledge Dr. Aldons J. Lusis for providing us with Lines 1 and 6 as well as Dr. David R. Beier (U01HD43430) and Dr. Jennifer Moran for conformational genotyping of these lines. We thank Dr. Katya Ravid, Dr. Kenneth Albrecht, and Greg Martin of the Boston University School of Medicine Transgenic and Genomic Engineering Core Facility (http:// www.bumc.bu.edu/transgenic/) who aided in generating TALENs-targeted mice.

\section{Author Contributions}

Conceived and designed the experiments: CDB AAP. Performed the experiments: NY CCP MAG LAK SLK JEL. Analyzed the data: NY CCP YS ERR GS RC WEJ. Contributed reagents/ materials/analysis tools: RC WEJ. Wrote the paper: CDB NY AAP JEL. Suggested additional analysis used in the supplementary information: JEL.

\section{References}

1. Goldman D, Oroszi G, Ducci F. (2005) The genetics of addictions: Uncovering the genes. Nat Rev Genet 6(7): 521-532. PMID: 15995696

2. Ho MK, Goldman D, Heinz A, Kaprio J, Kreek MJ, et al. (2010) Breaking barriers in the genomics and pharmacogenetics of drug addiction. Clin Pharmacol Ther 88(6): 779-791. doi: 10.1038/clpt.2010.175 PMID: 20981002

3. Gelernter J, Kranzler HR. (2010) Genetics of drug dependence. Dialogues Clin Neurosci 12(1): 77-84. PMID: 20373669

4. Bousman CA, Glatt SJ, Everall IP, Tsuang MT. (2009) Genetic association studies of methamphetamine use disorders: A systematic review and synthesis. Am J Med Genet B Neuropsychiatr Genet 150B(8): 1025-1049. doi: 10.1002/ajmg.b.30936 PMID: 19219857

5. Flint J, Timpson N, Munafo M. (2014) Assessing the utility of intermediate phenotypes for genetic mapping of psychiatric disease. Trends Neurosci 37(12): 733-741. doi: 10.1016/j.tins.2014.08.007 PMID: 25216981

6. Donaldson ZR, Hen R. (2014) From psychiatric disorders to animal models: A bidirectional and dimensional approach. Biol Psychiatry. S0006-3223(14)00098-5 [pii]. doi: 10.1016/j.biopsych.2014.10.028 PMID: 25842201

7. Vollm BA, de Araujo IE, Cowen PJ, Rolls ET, Kringelbach ML, et al. (2004) Methamphetamine activates reward circuitry in drug naive human subjects. Neuropsychopharmacology 29(9): 1715-1722. PMID: 15138439

8. Caligiuri MP, Buitenhuys C. (2005) Do preclinical findings of methamphetamine-induced motor abnormalities translate to an observable clinical phenotype? Neuropsychopharmacology 30(12): 21252134. 1300859 [pii]. PMID: 16123755

9. Fleckenstein AE, Volz TJ, Riddle EL, Gibb JW, Hanson GR. (2007) New insights into the mechanism of action of amphetamines. Annu Rev Pharmacol Toxicol 47: 681-698. PMID: 17209801

10. Howell LL, Negus SS. (2014) Monoamine transporter inhibitors and substrates as treatments for stimulant abuse. Adv Pharmacol 69: 129-176. doi: 10.1016/B978-0-12-420118-7.00004-4 PMID: 24484977 
11. Lominac KD, McKenna CL, Schwartz LM, Ruiz PN, Wroten MG, et al. (2014) Mesocorticolimbic monoamine correlates of methamphetamine sensitization and motivation. Front Syst Neurosci 8: 70. doi: 10. 3389/fnsys.2014.00070 PMID: 24847220

12. Palmer AA, Verbitsky M, Suresh R, Kamens HM, Reed CL, et al. (2005) Gene expression differences in mice divergently selected for methamphetamine sensitivity. Mamm Genome 16(5): 291-305. PMID: 16104378

13. Deminiere JM, Piazza PV, Le Moal M, Simon H. (1989) Experimental approach to individual vulnerability to psychostimulant addiction. Neurosci Biobehav Rev 13(2-3): 141-147. S0149-7634(89)80023-5 [pii]. PMID: 2682400

14. Schmidt KT, Weinshenker D. (2014) Adrenaline rush: The role of adrenergic receptors in stimulantinduced behaviors. Mol Pharmacol 85(4): 640-650. doi: 10.1124/mol.113.090118 PMID: 24499709

15. Arnsten AF. (2006) Stimulants: Therapeutic actions in ADHD. Neuropsychopharmacology 31(11): 2376-2383. 1301164 [pii]. PMID: 16855530

16. Mehler-Wex C, Riederer P, Gerlach M. (2006) Dopaminergic dysbalance in distinct basal ganglia neurocircuits: Implications for the pathophysiology of parkinson's disease, schizophrenia and attention deficit hyperactivity disorder. Neurotox Res 10(3-4): 167-179. PMID: 17197367

17. Hart AB, Gamazon ER, Engelhardt BE, Sklar P, Kahler AK, et al. (2014) Genetic variation associated with euphorigenic effects of $d$-amphetamine is associated with diminished risk for schizophrenia and attention deficit hyperactivity disorder. Proc Natl Acad Sci U S A 111(16): 5968-5973. doi: 10.1073/ pnas.1318810111 PMID: 24711425

18. Phillips TJ, Kamens HM, Wheeler JM. (2008) Behavioral genetic contributions to the study of addictionrelated amphetamine effects. Neuroscience and Biobehavioral Reviews 32(4): 707-59. doi: 10.1016/j. neubiorev.2007.10.008 PMID: 18207241

19. Bryant CD, Chang HP, Zhang J, Wiltshire T, Tarantino LM, et al. (2009) A major QTL on chromosome 11 influences psychostimulant and opioid sensitivity in mice. Genes Brain Behav 8(8): 795-805. doi: 10.1111/j.1601-183X.2009.00525.x PMID: 19694818

20. Parker CC, Cheng R, Sokoloff G, Palmer AA. (2012) Genome-wide association for methamphetamine sensitivity in an advanced intercross mouse line. Genes Brain Behav 11(1): 52-61. doi: 10.1111/j. 1601-183X.2011.00747.x PMID: 22032291

21. Cheng R, Lim JE, Samocha KE, Sokoloff G, Abney M, et al. (2010) Genome-wide association studies and the problem of relatedness among advanced intercross lines and other highly recombinant populations. Genetics 185(3): 1033-1044. doi: 10.1534/genetics.110.116863 PMID: 20439773

22. Bryant CD, Parker CC, Zhou L, Olker C, Chandrasekaran RY, et al. (2012) Csnk1e is a genetic regulator of sensitivity to psychostimulants and opioids. Neuropsychopharmacology 37(4): 1026-1035. doi: 10.1038/npp.2011.287 PMID: 22089318

23. Bryant CD, Kole LA, Guido MA, Sokoloff G, Palmer AA. (2012) Congenic dissection of a major QTL for methamphetamine sensitivity implicates epistasis. Genes Brain Behav 11(5): 623-632. doi: 10.1111/j. 1601-183X.2012.00795.x PMID: 22487465

24. Grisel JE, Belknap JK, O'Toole LA, Helms ML, Wenger CD, et al. (1997) Quantitative trait loci affecting methamphetamine responses in BXD recombinant inbred mouse strains. J Neurosci 17(2): 745-54. PMID: 8987796

25. SNELL GD, BUNKER HP. (1965) Histocompatibility genes of mice. V. five new histocompatibility loci identified by congenic resistant lines on a C57b 10 background. Transplantation 3: 235-252. PMID: 14265497

26. Shirley RL, Walter NA, Reilly MT, Fehr C, Buck KJ. (2004) Mpdz is a quantitative trait gene for drug withdrawal seizures. Nature Neuroscience 7(7): 699-700. PMID: 15208631

27. Tomida S, Mamiya T, Sakamaki H, Miura M, Aosaki T, et al. (2009) Usp46 is a quantitative trait gene regulating mouse immobile behavior in the tail suspension and forced swimming tests. Nat Genet 41 (6): 688-695. doi: 10.1038/ng.344 PMID: 19465912

28. lakoubova OA, Olsson CL, Dains KM, Ross DA, Andalibi A, et al. (2001) Genome-tagged mice (GTM): Two sets of genome-wide congenic strains. Genomics 74(1): 89-104. PMID: 11374905

29. Gold LH, Geyer MA, Koob GF. (1989) Neurochemical mechanisms involved in behavioral effects of amphetamines and related designer drugs. NIDA Res Monogr 94: 101-126. PMID: 2514360

30. Chesler EJ, Lu L, Wang J, Williams RW, Manly KF. (2004) WebQTL: Rapid exploratory analysis of gene expression and genetic networks for brain and behavior. Nature Neuroscience 7(5): 485-6. PMID: 15114364

31. Wefers B, Panda SK, Ortiz O, Brandl C, Hensler S, et al. (2013) Generation of targeted mouse mutants by embryo microinjection of TALEN mRNA. Nat Protoc 8(12): 2355-2379. doi: 10.1038/nprot.2013. 142 PMID: 24177293 
32. Wang J, Duncan D, Shi Z, Zhang B. (2013) WEB-based GEne SeT AnaLysis toolkit (WebGestalt): Update 2013. Nucleic Acids Res 41(Web Server issue): W77-83. doi: 10.1093/nar/gkt439 PMID: 23703215

33. Zhang B, Kirov S, Snoddy J. (2005) WebGestalt: An integrated system for exploring gene sets in various biological contexts. Nucleic Acids Res 33(Web Server issue): W741-8. 33/suppl_2/W741 [pii]. PMID: 15980575

34. Lein ES, Hawrylycz MJ, Ao N, Ayres M, Bensinger A, et al. (2007) Genome-wide atlas of gene expression in the adult mouse brain. Nature 445(7124): 168-176. nature05453 [pii]. PMID: 17151600

35. Yalcin B, Willis-Owen SA, Fullerton J, Meesaq A, Deacon RM, et al. (2004) Genetic dissection of a behavioral quantitative trait locus shows that Rgs2 modulates anxiety in mice. Nat Genet 36(11): 1197-1202. PMID: 15489855

36. Alavian KN, Jeddi S, Naghipour SI, Nabili P, Licznerski P, et al. (2014) The lifelong maintenance of mesencephalic dopaminergic neurons by Nurr1 and engrailed. J Biomed Sci 21: 27-0127-21-27. doi: 10.1186/s12929-014-0105-z PMID: 25547987

37. Drouin C, Darracq L, Trovero F, Blanc G, Glowinski J, et al. (2002) Alpha1b-adrenergic receptors control locomotor and rewarding effects of psychostimulants and opiates. J Neurosci 22(7): 2873-2884. PMID: 11923452

38. Sadalge A, Coughlin L, Fu H, Wang B, Valladares O, et al. (2003) Alpha 1d adrenoceptor signaling is required for stimulus induced locomotor activity. Mol Psychiatry 8(7): 664-672. PMID: 12874602

39. Dela Pena I, Jeon SJ, Lee E, Ryu JH, Shin CY, et al. (2013) Neuronal development genes are key elements mediating the reinforcing effects of methamphetamine, amphetamine, and methylphenidate. Psychopharmacology (Berl) 230(3): 399-413.

40. Le Merrer J, Befort K, Gardon O, Filliol D, Darcq E, et al. (2012) Protracted abstinence from distinct drugs of abuse shows regulation of a common gene network. Addict Biol 17(1): 1-12. doi: 10.1111/j. 1369-1600.2011.00365.x PMID: 21955143

41. Song KY, Choi HS, Law PY, Wei LN, Loh HH. (2012) Post-transcriptional regulation of mu-opioid receptor: Role of the RNA-binding proteins heterogeneous nuclear ribonucleoprotein $\mathrm{H} 1$ and $\mathrm{F}$. Cell Mol Life Sci 69(4): 599-610. doi: 10.1007/s00018-011-0761-z PMID: 21739230

42. Xu J, Xu M, Hurd YL, Pasternak GW, Pan YX. (2009) Isolation and characterization of new exon 11associated $\mathrm{N}$-terminal splice variants of the human mu opioid receptor gene. J Neurochem 108(4): 962-972. doi: 10.1111/j.1471-4159.2008.05833.x PMID: 19077058

43. Xu J, Lu Z, Xu M, Pan L, Deng Y, et al. (2014) A heroin addiction severity-associated intronic single nucleotide polymorphism modulates alternative pre-mRNA splicing of the mu opioid receptor gene OPRM1 via hnRNPH interactions. J Neurosci 34(33): 11048-11066. doi: 10.1523/JNEUROSCI.398613.2014 PMID: 25122903

44. Zheng X, Valakh V, Diantonio A, Ben-Shahar Y. (2014) Natural antisense transcripts regulate the neuronal stress response and excitability. Elife 3: e01849. doi: 10.7554/eLife.01849 PMID: 24642409

45. Han SP, Tang YH, Smith R. (2010) Functional diversity of the hnRNPs: Past, present and perspectives. Biochem J 430(3): 379-392. doi: 10.1042/BJ20100396 PMID: 20795951

46. Huelga SC, Vu AQ, Arnold JD, Liang TY, Liu PP, et al. (2012) Integrative genome-wide analysis reveals cooperative regulation of alternative splicing by hnRNP proteins. Cell Rep 1(2): 167-178. PMID: 22574288

47. Katz Y, Wang ET, Airoldi EM, Burge CB. (2010) Analysis and design of RNA sequencing experiments for identifying isoform regulation. Nat Methods 7(12): 1009-1015. doi: 10.1038/nmeth.1528 PMID: 21057496

48. Sinnamon JR, Czaplinski K. (2011) mRNA trafficking and local translation: The yin and yang of regulating mRNA localization in neurons. Acta Biochim Biophys Sin (Shanghai) 43(9): 663-670.

49. Zhang G, Neubert TA, Jordan BA. (2012) RNA binding proteins accumulate at the postsynaptic density with synaptic activity. J Neurosci 32(2): 599-609. doi: 10.1523/JNEUROSCI.2463-11.2012 PMID: 22238095

50. Van Dusen CM, Yee L, McNally LM, McNally MT. (2010) A glycine-rich domain of hnRNP H/F promotes nucleocytoplasmic shuttling and nuclear import through an interaction with transportin 1. Mol Cell Biol 30(10): 2552-2562. doi: 10.1128/MCB.00230-09 PMID: 20308327

51. Milosevic J, Bulau P, Mortz E, Eickelberg O. (2009) Subcellular fractionation of TGF-beta1-stimulated lung epithelial cells: A novel proteomic approach for identifying signaling intermediates. Proteomics 9 (5): 1230-1240. doi: 10.1002/pmic.200700604 PMID: 19253281

52. Keane TM, Goodstadt L, Danecek P, White MA, Wong K, et al. (2011) Mouse genomic variation and its effect on phenotypes and gene regulation. Nature 477(7364): 289-294. doi: 10.1038/nature10413 PMID: 21921910 
53. Yalcin B, Wong K, Agam A, Goodson M, Keane TM, et al. (2011) Sequence-based characterization of structural variation in the mouse genome. Nature 477(7364): 326-329. doi: 10.1038/nature10432 PMID: 21921916

54. Chorev M, Carmel L. (2012) The function of introns. Front Genet 3: 55. doi: 10.3389/fgene.2012.00055 PMID: 22518112

55. Glatt SJ, Cohen OS, Faraone SV, Tsuang MT. (2011) Dysfunctional gene splicing as a potential contributor to neuropsychiatric disorders. Am J Med Genet B Neuropsychiatr Genet 156B(4): 382-392. doi: 10.1002/ajmg.b.31181 PMID: 21438146

56. Fogel BL, Wexler E, Wahnich A, Friedrich T, Vijayendran C, et al. (2012) RBFOX1 regulates both splicing and transcriptional networks in human neuronal development. Hum Mol Genet 21(19): 4171-4186. doi: 10.1093/hmg/dds240 PMID: 22730494

57. Sun S, Zhang Z, Fregoso O, Krainer AR. (2012) Mechanisms of activation and repression by the alternative splicing factors RBFOX1/2. RNA 18(2): 274-283. doi: 10.1261/rna.030486.111 PMID: 22184459

58. Bill BR, Lowe JK, Dybuncio CT, Fogel BL. (2013) Orchestration of neurodevelopmental programs by RBFOX1: Implications for autism spectrum disorder. Int Rev Neurobiol 113: 251-267. doi: 10.1016/ B978-0-12-418700-9.00008-3 PMID: 24290388

59. Pascale A, Amadio M, Quattrone A. (2008) Defining a neuron: Neuronal ELAV proteins. Cell Mol Life Sci 65(1): 128-140. PMID: 17928954

60. Kim HJ, Raphael AR, LaDow ES, McGurk L, Weber RA, et al. (2014) Therapeutic modulation of elF2alpha phosphorylation rescues TDP-43 toxicity in amyotrophic lateral sclerosis disease models. Nat Genet 46(2): 152-160. doi: 10.1038/ng.2853 PMID: 24336168

61. Cheng R, Abney M, Palmer AA, Skol AD. (2011) QTLRel: An R package for genome-wide association studies in which relatedness is a concern. BMC Genet 12: 66. doi: 10.1186/1471-2156-12-66 PMID: 21794153

62. Cheng R, Palmer AA. (2013) A simulation study of permutation, bootstrap, and gene dropping for assessing statistical significance in the case of unequal relatedness. Genetics 193(3): 1015-1018. doi: 10.1534/genetics.112.146332; PMID: 23267053

63. Belknap JK. (2003) Chromosome substitution strains: Some quantitative considerations for genome scans and fine mapping. Mamm Genome 14(11): 723-32. PMID: 14722722

64. Nadeau JH, Singer JB, Matin A, Lander ES. (2000) Analysing complex genetic traits with chromosome substitution strains. Nature Genetics 24(3): 221-5. PMID: 10700173

65. Trapnell C, Roberts A, Goff L, Pertea G, Kim D, et al. (2012) Differential gene and transcript expression analysis of RNA-seq experiments with TopHat and cufflinks. Nat Protoc 7(3): 562-578. doi: 10.1038/ nprot.2012.016; PMID: 22383036

66. Robinson MD, McCarthy DJ, Smyth GK. (2010) edgeR: A bioconductor package for differential expression analysis of digital gene expression data. Bioinformatics 26(1): 139-140. doi: 10.1093/ bioinformatics/btp616 PMID: 19910308

67. Benjamini Y, Hochberg Y. (1995) Controlling false discovery rate: A practical and powerful approach to multiple testing. Journal of the Royal Statistical Society 57(1): 289-300.

68. Schmittgen TD, Livak KJ. (2008) Analyzing real-time PCR data by the comparative C(T) method. Nature Protocols 3(6): 1101-8. PMID: 18546601

69. Kramer A, Green J, Pollard J Jr, Tugendreich S. (2014) Causal analysis approaches in ingenuity pathway analysis. Bioinformatics 30(4): 523-530. doi: 10.1093/bioinformatics/btt703 PMID: 24336805 